\title{
Morrey Estimates for Parabolic Nondivergence Operators of Hörmander Type
}

\author{
Sufang Tang - Pengcheng Niu (*)
}

Abstract - Let $X_{1}, X_{2}, \cdots, X_{q}$ be a system of real smooth vector fields satisfying Hörmander's rank condition in a bounded domain $\Omega$ of $\mathbb{R}^{n}$. Let $a_{i j}(t, x)$ be real valued, bounded measurable functions defined in a bounded domain $U \subset \mathbb{R} \times \Omega$, satisfying the uniform ellipticity condition:

$$
\mu^{-1}|\xi|^{2} \leq \sum_{i, j=1}^{q} a_{i j}(t, x) \xi_{i} \xi_{j} \leq \mu|\xi|^{2}
$$

for $\xi \in \mathbb{R}^{q}$ and some constant $\mu>0$. Moreover, we assume that the coefficients $a_{i j}$ belong to the space VMO ("Vanishing Mean Oscillation") with respect to the subelliptic metric induced by the vector fields $X_{1}, X_{2} \cdots X_{q}$. For parabolic nondivergence operator

$$
H_{1}=\partial_{t}-\sum_{i, j=1}^{q} a_{i j}(t, x) X_{i} X_{j}-\sum_{j=1}^{q} b_{j}(t, x) X_{j}-c(t, x),
$$

we prove the following local estimate:

$$
\|f\|_{S_{X}^{k+2, p, \lambda}\left(U^{\prime}\right)} \leq c\left\{\left\|H_{1} f\right\|_{S_{X}^{k, p, \lambda}(U)}+\|f\|_{L^{p, \lambda}(U)}\right\},
$$

in Sobolev-Morrey spaces $S_{X}^{k+2, p, \lambda}(U)$ defined by the vector fields $X_{i}$, where $f \in S_{X}^{k+2, p, \lambda}(U), H_{1} f \in S_{X}^{k, p, \lambda}(U), U^{\prime} \subset \subset U$, and $b_{j}, c$ belong to suitable Morrey spaces.

(*) Indirizzo degli A.: Department of Applied Mathematics, Northwestern Polytechnical University, 710072, Xi'an, Shaanxi, P.R. China

E-mail: sufangtang@163.com E-mail: pengchengniu@nwpu.edu.cn

Corresponding author: Pengcheng Niu

This work was supported by Specialized Research Fund for the Doctoral Program of Higher Education (Grant No. 200806990032), the National Natural Science Foundation of China (Grant No. 10871157) and Keji Chuangxin Jijin of Northwestern Polytechnical University (Grant No. 2008KJ02033)

2000 Mathematics Subject Classification. 35R05, 35B45, 42B20. 


\section{Introduction}

Let $X_{1}, X_{2} \cdots X_{q}$ be a system of $C^{\infty}$ real vector fields defined in $\mathbb{R}^{n}(n \geq q+1)$, with the form

$$
X_{i}=\sum_{j=1}^{n} b_{i j}(x) \frac{\partial}{\partial x_{j}}, \text { with } b_{i j} \in C^{\infty}\left(\mathbb{R}^{n}\right),
$$

$(i=1, \cdots q ; j=1,2, \cdots n)$ and $\left\{X_{i}\right\}$ satisfy the so-called "Hörmander's condition" at every point of $\Omega \subset \mathbb{R}^{n}$ : the vector fields $X_{i}$, their commutators $\left[X_{i}, X_{j}\right]=X_{i} X_{j}-X_{j} X_{i}$, the commutators of the $X_{i}$ 's with their commutators, and so on until a certain step $s$, generate $\mathbb{R}^{n}$ (as a vector space). In this setting, "sum of squares" operators

$$
\sum_{i=1}^{q} X_{i}^{2}
$$

or their "parabolic" analog

$$
\partial_{t}-\sum_{i=1}^{q} X_{i}^{2}
$$

are hypoelliptic in $\Omega$ or $U \subset \mathbb{R} \times \Omega([2,20])$. In recent years, more general operators

$$
\sum_{i, j=1}^{q} a_{i j}(x) X_{i} X_{j}
$$

and

$$
\partial_{t}-\sum_{i, j=1}^{q} a_{i j}(t, x) X_{i} X_{j}
$$

have been studied, where $a_{i j}$ satisfy the uniform ellipticity condition and belong to VMO ("Vanishing Mean Oscillation", see Definition 2.3 below). These classes of operators come from geometry in several complex variables and human vision; moreover, they offer a suitable framework for studying the theory of nonlinear equations modeled on vector fields(see [2] and references therein).

For second-order nondivergence elliptic operators, Chiarenza, Frasca and Longo [10-11] first utilized the $L^{p}$ boundedness of singular integral operators with variable kernels and their commutators with functions in $B M O$ ("Bounded Mean Oscillation") to prove the $L^{p}$ estimates of the so- 
lutions; applying the same technique, Bramanti and Cerutti [5] obtained similar results concerning second-order nondivergence parabolic operators; the estimates in Morrey spaces on the Euclidean space for secondorder nondivergence elliptic operators were established by Di Fazio, Palagachev, Ragusa [13-14] through adapting the approach from [10]; for second-order nondivergence elliptic and parabolic operators, Lieberman [21] derived directly the estimates in Morrey spaces from the $L^{p}$ estimates via an elementary argument.

Rothschild and Stein [24] put forward the technique of "lifting and approximation", and proved $L^{p}$ estimates of the operator (1.3) with $a_{i j} \in C^{\infty}$. Using the idea in [24], Bramanti and Brandolini established $L^{p}$ estimates of the operator (1.3) in [1], and Schauder estimates of the operator (1.4) and more general parabolic operators in [2], respectively. From the view of analysis, we think that it is interesting and important to study regularities in various function spaces for these operators constructed by Hörmander's vector fields.

Our aim is to check local Morrey estimates of the operator

$$
H_{1}=\partial_{t}-\sum_{i, j=1}^{q} a_{i j}(t, x) X_{i} X_{j}-\sum_{j=1}^{q} b_{j}(t, x) X_{j}-c(t, x) .
$$

The research of this paper explores new efficacy and applications of the technique by Rothschild and Stein and new properties of singular integrals on homogeneous spaces.

We are now in position to list our main assumptions:

(H1) Let $\Omega$ be a bounded domain of $\mathbb{R}^{n}$, and let $X_{1}, X_{2}, \cdots, X_{q}$ be a system of smooth real vector fields defined in a neighborhood $\Omega_{0}$ of $\Omega$ and satisfy Hörmander's condition of step $s$ in $\Omega_{0}$.

(H2) Let $U$ be a bounded domain of $\mathbb{R}^{n+1}, U \subset \mathbb{R} \times \Omega$ and let $a_{i j}(t, x)$ be real valued bounded measurable functions defined in $U$; there exists some constant $\mu>0$ such that the matrix $\left\{a_{i j}(t, x)\right\}_{i, j=1}^{q}$ (not necessarily symmetric) satisfies the uniformly ellipticity condition:

$$
\mu^{-1}|\xi|^{2} \leq \sum_{i, j=1}^{q} a_{i j}(t, x) \xi_{i} \xi_{j} \leq \mu|\xi|^{2}, \text { for every } \xi \in \mathbb{R}^{q},(t, x) \in U .
$$

(H3) Let $a_{i j}(t, x)$ belong to the class $V M O(U)$ defined with respect to the subelliptic metric induced by $X_{1}, X_{2} \cdots X_{q}$. 
We will see that $U$ becomes a homogeneous space with respect to a Carnot-Caratheodory distance(see Section 5 below). Then there exists a positive integer $q^{\prime}$ such that the measure $\mu\left(B_{r}\right)$ of the ball $B_{r}$ with respect to the C-C distance in $\mathbb{R}^{n+1}$ is equivalent to $r q^{\prime}$.

Our main result is the following:

Theorem 1.1. Assume that (H1)-(H3) hold, $a_{i j}(t, x) \in S_{X}^{k, \infty, 0}(U)$ and $b_{j}(t, x), c(t, x)$ in (1.5) satisfy the following:

$b_{j} \in\left\{\begin{array}{l}L^{q^{\prime}} \cap S_{X}^{k, q^{\prime}, 0}(U), p+\lambda \leq q^{\prime} ; \\ L^{p, \lambda} \cap S_{X}^{k, p, \lambda}(U), p+\lambda>q^{\prime},\end{array} \quad c \in\left\{\begin{array}{l}L^{q^{\prime} / 2}(U) \cap S_{X}^{k, q^{\prime} / 2,0}(U), 2 p+\lambda \leq q^{\prime} ; \\ L^{p, \lambda}(U) \cap S_{X}^{k, p, \lambda}(U), 2 p+\lambda>q^{\prime},\end{array}\right.\right.$

for every $p \in(1,+\infty)$ and $\lambda \in\left(0, q^{\prime}\right)$, where $L^{p, \lambda}$ denotes some Morrey spaces (see Section 2), $S_{X}^{k, q^{\prime}, 0}, S_{X}^{k, q^{\prime} / 2,0}, S_{X}^{k, p, \lambda}$ are Sobolev-Morrey spaces(see Section 7). Then for every subdomain $U^{\prime} \subset \subset U$, there exists a constant $C>0$ depending on $U, U^{\prime},\left\{X_{i}\right\}, p, \lambda, \mu$ and suitable norms of the coefficients $a_{i j}, b_{j}, c$ such that for every $f \in S_{X}^{k+2, p, \lambda}(U)$ with $H_{1} f \in S_{X}^{k, p, \lambda}(U)$ and $k \in \mathbb{N}$, we have

$$
\|f\|_{S_{X}^{k+2, p, \lambda}\left(U^{\prime}\right)} \leq C\left\{\left\|H_{1} f\right\|_{S_{X}^{k, p, \lambda}(U)}+\|f\|_{L^{p, \lambda}(U)}\right\} .
$$

REMARK 1.2. Analogous Morrey estimates for the operator (1.3) obviously follow from Theorem 1.1. Moreover, $L^{p}$ estimates of (1.3) (i.e. Theorem 0.1 and Theorem 0.2 in [1]) are promptly derived, as a particular case.

We will use "lifting and approximation" and the Morrey boundedness of variable operators of type 0 and their commutators with $B M O$ functions in the proof of Theorem 1.1. Let us note that the Morrey boundedness of singular integrals and their commutators with $B M O$ functions on homogeneous spaces was established in [16]. Here we prove the Morrey boundedness of fractional integrals and their commutators (Theorem 3.2 below) and interpolation theorem in the Morrey context (Theorem 3.9 below). They play a crucial role in the proof of properties of variable operators of type 0 , and are of independent interest.

Compared with the existing literature, we do not see any work on the study of Morrey regularities for solutions of (1.5) involving (1.3) and (1.4).

The paper is organized as follows: In Section 2 we introduce definitions of $B M O, V M O$ and Morrey space on homogeneous spaces; Section 3 is devoted to the Morrey boundedness of fractional integrals and their 
commutators with $B M O$ functions, and the interpolation theorem with respect to Morrey norm; In Section 4 we first recall the "lifting and approximation" technique ([24]). For our purpose, we define a homogeneous group by a natural way and a quasidistance on the group; In Section 5 we give some properties of "parabolic Carnot-Caratheodory distance" induced by Hörmander's vector fields, and their relations with the quasidistance on homogeneous groups; In Section 6, collecting some known results about the fundamental solution to the frozen lifted operators (see [15]) and some properties of the frozen and variable operators of type $l \geq 0([1,24])$, we prove the Morrey boundedness of variable operators of type 0 and their commutators with BMO functions using the theory of Section 3. It is important in the argument of the following sections; The main tasks in Section 7, 8 are to prove local estimates of the operator (1.5) without lowerorder terms in Sobolev-Morrey spaces of $S_{X}^{2, p, \lambda}(U)$ and $S_{X}^{k+2, p, \lambda}(U)$, respectively; In Section 9, results of Section 7 and 8 are extended to (1.5) and then the proof of Theorem 1.1 is completed.

\section{Some function spaces.}

Let $S$ be a set. A function $d: S \times S \rightarrow \mathbb{R}$ is called a quasidistance, if there exists a constant $c \geq 1$ such that for any $x, y, z \in S$,

$$
\left\{\begin{array}{l}
d(x, y) \geq 0, \quad d(x, y)=0 \text { if and only if } x=y \\
c^{-1} d(y, x) \leq d(x, y) \leq c d(y, x) \\
d(x, y) \leq c(d(x, z)+d(z, y))
\end{array}\right.
$$

We say that two quasidistances $d, d^{\prime}$ on $S$ are equivalent, if there exist two positive constants $c_{1}, c_{2}$ such that $c_{1} d^{\prime}(x, y) \leq d(x, y) \leq c_{2} d^{\prime}(x, y)$ for any $x, y \in S$. In this case, we write $d \simeq d^{\prime}$.

For $r>0$, let $B_{r}(x)=\{y \in S: d(x, y)<r\}$ be a ball in $S$.

Definition 2.1 (Homogeneous space, see Coifman-Weiss [12]). Let $(S, d)$ be a space endowed with a quasidistance d such that balls defined by d induce a topology in S. Moreover, we assume that $\mu$ is a positive Borel measure on $S$ satisfying the doubling condition, i.e., there exists a positive constant $c_{\mu}$ such that

$$
0<\mu\left(B_{2 r}(x)\right) \leq c_{\mu} \cdot \mu\left(B_{r}(x)\right)<\infty, \quad \text { for every } r>0, x \in S .
$$

Then $(S, d, \mu)$ is called a homogeneous space. 
To simplify notations, the measure $d \mu(x)$ will be written as $d x$, and $\mu(\Omega)$ as $|\Omega|$.

Definition 2.2. A homogeneous space is said to be regular, if there exist positive constants $c_{1}, c_{2}$ and $Q>0$ such that

$$
c_{1} r^{Q}<\mu\left(B_{r}(x)\right) \leq c_{2} r^{Q}
$$

for every $x \in S$ and $r, \mu(\{x\})<r \leq \mu(S)$.

From [22], we know that for every homogeneous space $(S, d, \mu)$, there exists a regular homogeneous space $\left(S, d^{\prime}, \mu\right)$ such that $d$ and $d^{\prime}$ are equivalent.

Next we introduce $B M O, V M O$ and Morrey space.

Definition 2.3. For a measurable function $f \in L_{\text {loc }}^{1}(S)$, define

$$
\eta_{f}(R)=\sup _{r \leq R} \oint_{B_{r}}\left|f(x)-f_{B_{r}}\right| d x
$$

where $f_{B_{r}}=\oint_{B_{r}} f(x) d x=\frac{1}{\left|B_{r}\right|} \int_{B_{r}} f(x) d x$ Then $f \in B M O(S)$ (Bounded Mean Oscillation) if $\|f\|_{*}:=\sup _{R} \eta_{f}(R)<+\infty$, while $f \in V M O(S)$ (Vanishing Mean Oscillation) if $\lim _{R \rightarrow 0} \eta_{f}(R)=0$.

For a given domain $\Omega \subset S$, the spaces $B M O(\Omega)$ and $\operatorname{VMO}(\Omega)$ are similarly defined, just taking $B_{r} \cap \Omega$ instead of $B_{r}$.

DEFINITION 2.4. We say that a measurable function $f \in L_{l o c}^{1}(S)$ belongs to the Morrey space $L^{p, \lambda}(S)$ with $p \in(1,+\infty)$ and $\lambda \in(0, Q)$, if the following norm

$$
\|f\|_{p, \lambda}:=\left(\sup _{r>0} \frac{1}{r^{\lambda}} \int_{B_{r}}|f(x)|^{p} d x\right)^{1 / p}
$$

is finite.

Similarly, the space $L^{p, \lambda}(\Omega)$ and the norm $\|f\|_{p, \lambda ; \Omega}$ are defined with some explicit changes. 


\section{Singular integrals and interpolation theorem.}

For a linear operator $T$ on $L^{p}(S)$, the commutator of $T$ and $a \in B M O(S)$ is defined by

$$
[T, a] f=T(a f)-a T f .
$$

$T$ is called a sublinear operator from $L^{p, \lambda}(\Omega)$ to $L^{q, \mu}(\Omega)$ for $p, q \in(1,+\infty)$ and $\lambda, \mu \in(0, Q)$, if there exists a constant $K>0$ such that

$$
|T(f+g)(x)| \leq K(|T f(x)|+|T g(x)|), \quad \forall f, g \in L^{p, \lambda}(\Omega), \text { a.e. } \Omega .
$$

LEMma 3.1 (Singular integral and commutator, [16]). Let $(S, d, \mu)$ be a regular homogeneous space, $p \in(1,+\infty)$ and $\lambda \in(0, Q)$. Assume that a sublinear operator $T$ satisfies the following: for every function $f \in L^{1}(S)$ with compact support and every $x \notin \operatorname{sprt} f$,

$$
|T f(x)| \leq C \int_{S} \frac{|f(y)|}{d(x, y)^{Q}} d y .
$$

(i) If $T$ is bounded from $L^{p}(S)$ into itself, then $T$ is also bounded from $L^{p, \lambda}(S)$ into itself, that is,

$$
\|T f\|_{p, \lambda} \leq C\|f\|_{p, \lambda} ;
$$

(ii) If $[T, a]$ is bounded from $L^{p}(S)$ into itself, then $[T, a]$ is also bounded from $L^{p, \lambda}(S)$ into itself, that is,

$$
\|[T, a](f)\|_{p, \lambda} \leq C\|a\|_{*}\|f\|_{p, \lambda} .
$$

Theorem 3.2 (Fractional integral and commutator). Let $(S, d, \mu)$ be a regular homogeneous space, $\alpha, \lambda \in(0, Q)$ and $\lambda+p \alpha<Q$. Let $T$ satisfy

$$
|T f(x)| \leq C \int_{S} \frac{|f(y)|}{d(x, y)^{Q-\alpha}} d y,
$$

for every function $f \in L^{1}(S)$ with compact support and every $x \notin \operatorname{sprt} f$, and denote $\frac{1}{q}=\frac{1}{p}-\frac{\alpha}{Q}, \mu=\frac{\lambda}{p} q$. Then we have

(i) if $T$ is bounded from $L^{p}(S)$ into $L^{q}(S)$, then $T$ is bounded from $L^{p, \lambda}(S)$ to $L^{q, \mu}(S)$, that is,

$$
\|T f\|_{q, \mu} \leq C\|f\|_{p, \lambda}
$$


(ii) if $[T, a]$ is bounded from $L^{p}(S)$ into $L^{q}(S)$, then $[T, a]$ is also bounded from $L^{p, \lambda}(S)$ to $L^{q, \mu}(S)$, that is,

$$
\|[T, a] f\|_{q, \mu} \leq C\|a\|_{*}\|f\|_{p, \lambda} .
$$

Proof. (i) As in [16], for any $x_{0} \in S, r>0, f$ can be decomposed into

$$
f(y)=f \chi_{B_{2 r}\left(x_{0}\right)}(y)+\sum_{k=1}^{\infty} f \chi_{B_{2^{k+1} r_{r}}\left(x_{0}\right) \backslash B_{2^{k}}\left(x_{0}\right)}(y):=f_{0}(y)+\sum_{k=1}^{\infty} f_{k}(y) .
$$

Hence,

(3.8) $\left(\int_{B_{r}\left(x_{0}\right)}|T f(x)|^{q} d x\right)^{1 / q} \leq\left(\int_{B_{r}\left(x_{0}\right)}\left|T f_{0}(x)\right|^{q} d x\right)^{1 / q}+\sum_{k=1}^{\infty}\left(\int_{B_{r}\left(x_{0}\right)}\left|T f_{k}(x)\right|^{q} d x\right)^{1 / q}$.

Using the boundedness of $T$ from $L^{p}(S)$ to $L^{q}(S)$ yields

$$
\left(\int_{B_{r}\left(x_{0}\right)}\left|T f_{0}(x)\right|^{q} d x\right)^{1 / q} \leq C\left(\int_{B_{r}\left(x_{0}\right)}\left|f_{0}(x)\right|^{p} d x\right)^{1 / p} \leq C r^{\lambda / p}\|f\|_{p, \lambda},
$$

and for $k \geq 1$,

$$
\left(\int_{B_{r}\left(x_{0}\right)}\left|T f_{k}(x)\right|^{q} d x\right)^{1 / q} \leq C\left(\int_{B_{r}\left(x_{0}\right)}\left(\int_{B_{2^{k+1}}\left(x_{0}\right) \backslash B_{2^{k}}\left(x_{0}\right)} \frac{|f(y)|}{d(x, y)^{Q-\alpha}} d y\right)^{q} d x\right)^{1 / q}
$$

$$
\begin{aligned}
& \leq C \frac{r^{Q / q}}{\left(2^{k} r\right)^{Q-\alpha}} \int_{B_{2^{k+1}}\left(x_{0}\right)}|f(y)| d y \\
& \leq C \frac{1}{2^{k((Q-\lambda) / p-\alpha)}} r^{\lambda / p}\|f\|_{p, \lambda}
\end{aligned}
$$

(3.6) is immediately obtained from (3.8)-(3.10).

(ii) As in the proof of (i), let

$$
f(y)=: f_{0}(y)+\sum_{k=1}^{\infty} f_{k}(y) .
$$

Since $[T, a]$ is bounded from $L^{p}(X)$ to $L^{q}(X)$, one has

$$
\left(\int_{B_{r}\left(x_{0}\right)}\left|[T, a] f_{0}(x)\right|^{q} d x\right)^{1 / q} \leq C\|a\|_{*}\left\|f_{0}\right\|_{p} \leq C r^{\lambda / p}\|a\|_{*}\|f\|_{p, \lambda} .
$$


For every $\sigma>0$, set

$$
a_{B_{\sigma}}=\frac{1}{\left|B_{\sigma}\left(x_{0}\right)\right|} \int_{B_{\sigma}\left(x_{0}\right)} a(y) d y .
$$

John-Nirenberg lemma and Lemma 1.8 in [6] imply

$$
\begin{aligned}
& \left(\int_{B_{r}\left(x_{0}\right)}\left|[T, a] f_{k}(x)\right|^{q} d x\right)^{1 / q} \\
& \left.\leq C \int_{B_{r}\left(x_{0}\right)}\left(\int_{B_{2^{k+1}}\left(x_{0}\right) \backslash B_{2^{k}}\left(x_{0}\right)} \frac{\left|a(x)-a_{B_{r}}\right|+\left|a_{B_{r}}-a(y)\right|}{d(x, y)^{Q-\alpha}}\left|f_{k}(y)\right| d y\right)^{q} d x\right)^{1 / q} \\
& \leq C \frac{1}{\left(2^{k} r\right)^{Q-\alpha}}\left\{\left(\int_{B_{r}\left(x_{0}\right)}\left|a(x)-a_{B_{r}}\right|^{q} d x\right)^{1 / q} \int_{B_{2^{k+1}}\left(x_{0}\right)}|f(y)| d y\right. \\
& \text { (3.12) } \left.\quad+r^{Q / q} \int_{B_{2^{k+1} r_{r}\left(x_{0}\right)}}\left|a_{B_{r}}-a(y) \| f(y)\right| d y\right\} \\
& \leq C \frac{1}{\left(2^{k} r\right)^{Q-\alpha}}\left\{r^{Q / q}\|a\|_{*} \int_{B_{2^{k+1} r^{(}\left(x_{0}\right)}}|f(y)| d y\right. \\
& \left.+r^{Q / q}\left(\int_{B_{2^{k+1}}\left(x_{0}\right)}\left|a_{B_{r}}-a(y)\right|^{1 /(1-1 / p)} d y\right)^{1-1 / p}\left(\int_{B_{2^{k+1} 1_{r}\left(x_{0}\right)}}|f(y)|^{p} d y\right)^{1 / p}\right\} \\
& \leq C \frac{1}{2^{k((Q-\lambda) / p-\alpha)}} r^{\lambda / p}\|a\|_{*}\|f\|_{p, \lambda} \text {. }
\end{aligned}
$$

It is easy to derive (3.7) form (3.11)-(3.12).

Theorem 3.3 (Embedding property). Let $\Omega$ be a bounded open subset of $S$. If $q \leq p$ and $\frac{-Q+\mu}{q} \leq \frac{-Q+\lambda}{p}$, then $L^{p, \lambda}(\Omega)$ is embedded continuously into $L^{q, \mu}(\Omega)$, and we will write $L^{p, \lambda}(\Omega) \hookrightarrow L^{q, \mu}(\Omega)$.

It can be proved by Hölder's inequality.

Next we extend the interpolation theorem on Sobolev spaces established by Marcinkiewicz (see [9, Chapter 3] or [19, Chapter 9]) to the case of Morrey spaces. To do this, we need more precise estimates in the Morrey context. 
Definition 3.4. Let $f \in L^{1}(\Omega), x_{0} \in \Omega, r>0, t \geq 0$, and let

$$
A_{t, r}(f)=\left\{x \in \Omega \cap B_{r}\left(x_{0}\right)|| f(x) \mid>t\right\} .
$$

The function

$$
\lambda_{f}(t, r)=\left|A_{t, r}(f)\right|
$$

is called a distribution function of $f$.

Lemma 3.5 ([9, Chapter 3, Lemma 1.1] or [19, Lemma 9.7]). For $f \in L^{p}(\Omega)(1 \leq p<\infty)$, it holds

$$
\int_{B_{r}\left(x_{0}\right) \cap \Omega}|f(x)|^{p} d x=p \int_{0}^{\infty} t^{p-1}\left|A_{t, r}(f)\right| d t .
$$

Definition 3.6 (Weak Morrey space). For $p \in(1,+\infty), \lambda \in(0, Q), a$ measurable function $f$ is said to belong to weak $L^{p, \lambda}\left(\right.$ denoted by $\left.L_{w}^{p, \lambda}(\Omega)\right)$, if

$$
\|f\|_{L_{w}^{p, \lambda}(\Omega)}=\sup _{r>0} \inf \left\{A \mid \lambda_{f}(t, r) \leq r^{\lambda} t^{-p} A^{p}, \forall t>0, r>0\right\}<\infty .
$$

Lemma 3.7. For any $1<q<p<\infty, 0<\mu<\lambda<Q$, one has

$$
L^{p, \lambda}(\Omega) \varsubsetneqq L_{w}^{p, \lambda}(\Omega) \subset L^{q, \mu}(\Omega) .
$$

Proof. For any $f \in L^{p, \lambda}(\Omega)$,

$$
r^{-\lambda} t^{p}\left|A_{t, r}(f)\right| \leq r^{-\lambda} \int_{A_{t, r}}|f(x)|^{p} d x=r^{-\lambda} \int_{B_{r}\left(x_{0}\right) \cap \Omega}|f(x)|^{p} d x,
$$

and therefore, $\|f\|_{L_{w}^{p, \lambda}(\Omega)} \leq\|f\|_{L^{p, \lambda}(\Omega)}$.

If $f \in L_{w}^{p, \lambda}(\Omega)$, then by Lemma 3.5,

$$
\begin{aligned}
r^{-\mu} \int_{B_{r}\left(x_{0}\right) \cap \Omega}|f(x)|^{q} d x & =q r^{-\mu} \int_{0}^{1} t^{q-1}\left|A_{t, r}(f)\right| d t+q r^{-\mu} \int_{1}^{\infty} t^{q-1}\left|A_{t, r}(f)\right| d t \\
& =: I+I I .
\end{aligned}
$$

We have

$$
|I| \leq q r^{-\mu}\left|B_{r}\left(x_{0}\right) \cap \Omega\right| \int_{0}^{1} t^{q-1} d t \leq C r^{Q-\mu}, \quad \text { for } Q-\mu>0,
$$




$$
\begin{aligned}
|I I| & \leq q r^{-\mu}\left(\int_{1}^{\infty} t^{-1-\frac{p}{2}} t^{p}\left|A_{t, r}(f)\right| d t\right)^{\frac{p-q}{p}} \cdot\left(\int_{1}^{\infty} t^{-1-\frac{(p-q) p}{2 q}} t^{p}\left|A_{t, r}(f)\right| d t\right)^{\frac{q}{p}} \\
& \leq q r^{-\mu} r^{\frac{\lambda(p-q)}{p}}\left(\|f\|_{L_{w}^{p, \lambda}(\Omega)}\right)^{p-q}\left(\int_{1}^{\infty} t^{-1-\frac{p}{2}} d t\right)^{\frac{p-q}{p}} \cdot r^{\frac{\lambda q}{p}}\left(\|f\|_{L_{w}^{p, \lambda}(\Omega)}\right)^{q}\left(\int_{1}^{\infty} t^{-1-\frac{(p-q) p}{2 q}} d t\right)^{\frac{q}{p}} \\
& =q r^{\lambda-\mu}\|f\|_{L_{w}^{p, \lambda}(\Omega)}^{p} .
\end{aligned}
$$

Applying (3.19) and (3.20) to (3.18) and taking $\sup _{B_{r}\left(x_{0}\right) \cap \Omega}$ yield

$$
\|f\|_{q, \mu ; \Omega}^{q} \leq C+C^{\prime}\|f\|_{L_{w}^{p, \lambda}(\Omega)}^{p}<\infty .
$$

Definition 3.8 (Type of sublinear operator). A sublinear operator $T$ is said to be a strong $(p, \lambda ; q, \mu)$ type, if there exists a constant $C>0$ such that

$$
\|T f\|_{q, \mu ; \Omega} \leq C\|f\|_{p, \lambda ; \Omega}, \quad \forall f \in L^{p, \lambda}(\Omega),
$$

for $1<p, q<\infty$ and $0<\lambda, \mu<Q$. Denote $\|T\|_{(p, \lambda ; q, \mu)}=\sup _{\substack{f \in L^{p, \lambda}(\Omega) \\ f \neq 0}} \frac{\|T f\|_{q, \mu}}{\|f\|_{p, \lambda}}$.

We say that $T$ is said to be a weak $(p, \lambda ; q, \mu)$ type, if

$$
\|T f\|_{L_{w}^{q, \mu}(\Omega)} \leq C\|f\|_{p, \lambda ; \Omega}, \quad \forall f \in L^{p, \lambda}(\Omega) .
$$

From (3.17) we know that if $T$ is a strong $(p, \lambda ; q, \mu)$ type, then $T$ is a weak $(p, \lambda ; q, \mu)$ type.

THeOREM 3.9 (Interpolation theorem). Let $1<p<q<\infty, 0<\lambda<\mu<Q$. If the sublinear operator $T$ is both weak $(p, \lambda ; p, \lambda)$ type and weak $(q, \mu ; q, \mu)$ type, that is,

$$
\|T f\|_{L_{w}^{p, \lambda}(\Omega)} \leq B_{p, \lambda}\|f\|_{p, \lambda ; \Omega}, \quad \forall f \in L^{p, \lambda}(\Omega),
$$

and

$$
\|T f\|_{L_{w}^{q, \mu}(\Omega)} \leq B_{q, \mu}\|f\|_{q, \mu ; \Omega}, \quad \forall f \in L^{q, \mu}(\Omega),
$$

then for any $\rho, \kappa: p<\rho<q, \lambda<\kappa<\mu$, T is strong $(\rho, \kappa ; \rho, \kappa)$ type, and

$$
\|T\|_{(\rho, \kappa ; \rho, \kappa)} \leq C B_{p, \lambda}^{\theta} B_{q, \mu}^{1-\theta},
$$

where $\theta=\frac{p}{\rho} \frac{(q-\rho)}{(q-p)}$ and $C$ depends on $\rho, \kappa, p, \lambda, q, \mu, \Omega$ and $K$ in (3.1). 
Proof. For $f \in L^{\rho, \kappa}(\Omega)$ and $s \geq 0$, we write $f=f_{1}+f_{2}$, where

$$
f_{1}(x)= \begin{cases}0, & \text { for }|f(x)| \leq \gamma s, \\ f(x), & \text { for }|f(x)|>\gamma s\end{cases}
$$

for some positive number $\gamma$ to be fixed later. We claim that $f_{1} \in L^{p, \lambda}(\Omega)$ and $f_{2} \in L^{q, \mu}(\Omega)$. In fact, for $x_{0} \in \Omega$,

$$
\left\|f_{1}\right\|_{L^{p, \lambda}(\Omega)} \leq\|f\|_{L^{p, \lambda}(\Omega)} \leq C\|f\|_{L^{p, \kappa}(\Omega)}<\infty
$$

and

$$
r^{-\mu} \int_{\Omega \cap B_{r}\left(x_{0}\right)}\left|f_{2}(x)\right|^{q} d x \leq C r^{-\kappa} \int_{\Omega \cap B_{r}\left(x_{0}\right)}|f(x)|^{\rho} d x<\infty .
$$

Hence, by (3.1),

$$
\begin{aligned}
\left|\left\{x \in \Omega \cap B_{r}\left(x_{0}\right)|| T f(x) \mid>s\right\}\right| & \leq\left|\left\{x \in \Omega \cap B_{r}\left(x_{0}\right)|| T f_{1}(x) \mid>\frac{s}{2 K}\right\}\right| \\
& +\left|\left\{x \in \Omega \cap B_{r}\left(x_{0}\right)|| T f_{2}(x) \mid>\frac{s}{2 K}\right\}\right| .
\end{aligned}
$$

From (3.21)-(3.23) we obtain

$$
\begin{aligned}
r^{-\kappa} \lambda_{T f}(s, r) \leq & C\left(r^{-\lambda}\left|\left\{x \in \Omega \cap B_{r}\left(x_{0}\right)|| T f_{1}(x) \mid>\frac{s}{2 K}\right\}\right|\right. \\
& \left.+r^{-\mu}\left|\left\{x \in \Omega \cap B_{r}\left(x_{0}\right)|| T f_{2}(x) \mid>\frac{s}{2 K}\right\}\right|\right) \\
\leq & C\left(\frac{(2 K)^{p}\left\|T f_{1}\right\|_{p, \lambda ; \Omega}^{p}}{s^{p}}+\frac{(2 K)^{q}\left\|T f_{2}\right\|_{q, \mu ; \Omega}^{q}}{s^{q}}\right) \\
\leq & C\left(\frac{\left(2 K B_{p, \lambda}\right)^{p}\left\|f_{1}\right\|_{p, \lambda ; \Omega}^{p}}{s^{p}}+\frac{\left(2 K B_{q, \mu}\right)^{q}\left\|f_{2}\right\|_{q, \mu ; \Omega}^{q}}{s^{q}}\right)
\end{aligned}
$$

where $C$ is a constant only dependent of $\mu, \kappa$ and $\Omega$. Also by Lemma 3.5,

$$
\begin{gathered}
r^{-\kappa} \int_{B_{r}\left(x_{0}\right) \cap \Omega}|T f(x)|^{\rho} d x=\rho \int_{0}^{\infty} s^{\rho-1} r^{-\kappa} \lambda_{T f}(s, r) d s \\
\leq C\left(2 K B_{p, \lambda}\right)^{p} \int_{0}^{\infty} \rho s^{\rho-p-1} d s\left(r^{-\lambda} \int_{\{|f(x)|>\gamma s\} \cap B_{r}\left(x_{0}\right)}\left|f_{1}(x)\right|^{p} d x\right)
\end{gathered}
$$




$$
\begin{aligned}
& +C\left(2 K B_{q, \mu}\right)^{q} \int_{0}^{\infty} \rho s^{\rho-q-1} d s\left(r^{-\mu} \int_{\{|f(x)| \leq \gamma s\} \cap B_{r}\left(x_{0}\right)}\left|f_{2}(x)\right|^{q} d x\right) \\
= & C\left(2 K B_{p, \lambda}\right)^{p} \rho r^{-\lambda} \int_{\Omega \cap B_{r}\left(x_{0}\right)}|f(x)|^{p} d x \int_{0}^{|f(x)| / \gamma} s^{\rho-p-1} d s \\
& +C\left(2 K B_{q, \mu}\right)^{q} \rho r^{-\mu} \int_{\Omega \cap B_{r}\left(x_{0}\right)}|f(x)|^{q} d x \int_{f(x) \mid / \gamma}^{\infty} \frac{1}{s^{q-\rho+1}} d s \\
= & C \frac{\left(2 K B_{p, \lambda}\right)^{p} \rho}{\rho-p} \gamma^{p-\rho} r^{-\lambda} \int_{\Omega \cap B_{r}\left(x_{0}\right)}|f(x)|^{\rho} d x+C \frac{\left(2 K B_{q, \mu}\right)^{q} \rho}{q-\rho} \gamma^{q-\rho} r^{-\mu} \underset{\Omega \cap B_{r}\left(x_{0}\right)}{\int}|f(x)|^{\rho} d x .
\end{aligned}
$$

Taking $\sup _{B_{r}\left(x_{0}\right) \cap \Omega}$ on both sides concludes

$$
\|T f\|_{\rho, \xi ; \Omega}^{\rho} \leq C\left[\frac{\left(2 K B_{p, \lambda}\right)^{p} \rho}{\rho-p} \gamma^{p-\rho}+\frac{\left(2 K B_{q, \mu}\right)^{q} \rho}{q-\rho} \gamma^{q-\rho}\right]\|f\|_{\rho, r ; \Omega}^{\rho},
$$

where $C$ relies also on $\lambda$. Choosing $\gamma=\left(B_{p, \lambda}^{p} B_{q, \mu}^{-q}\right)^{1 /(q-p)}$ follows

$$
\|T\|_{(\rho, \kappa ; \rho, \kappa)} \leq C B_{p, \lambda}^{\theta} B_{q, \mu}^{1-\theta}
$$

where $C$ depends on $\rho, p, q, \lambda, \mu, \kappa, K$ and $\Omega$.

Corollary 3.10. For $1<p<q<\infty$ and $0<\lambda<\mu<Q$, if a sublinear operator $T$ is both strong $(p, \lambda ; p, \lambda)$ type and strong $(q, \mu ; q, \mu)$ type, then for any $\rho, \kappa: p<\rho<q, \lambda<\kappa<\mu$, $T$ is strong $(\rho, \kappa ; \rho, \kappa)$ type.

\section{Lifting and approximation technique.}

Assume that $X_{1}, \cdots, X_{q}$ are $C^{\infty}$ real vector fields on a domain $\Omega \subset \mathbb{R}^{n}$ and satisfy Hörmander's condition of step $s$ at some point $x_{0} \in \Omega$. We also write $N$ for the dimension of the free nilpotent Lie algebra of step $s$ with $n$ generators. Then in the light of "lifting theorem" by Rothschild and Stein in [24], there exist smooth functions $\lambda_{i l}(x, h) \quad\left(1 \leq i \leq q, n+1 \leq l \leq N, h=\left(h_{n+1}, \cdots, h_{N}\right)\right)$ defined in a neighborhood $\tilde{U}$ of $\xi_{0}=\left(x_{0}, 0\right) \in \Omega \times \mathbb{R}^{N-n}=\tilde{\Omega}$ such that the vector fields 
$\tilde{X}_{i}$ given by

$$
\tilde{X}_{i}=X_{i}+\sum_{l=n+1}^{N} \lambda_{i l}(x, h) \frac{\partial}{\partial h_{l}}, \quad i=1, \cdots q,
$$

also satisfy Hörmander's condition of step $s$ and are free up to step $s$ at every point in $\tilde{U}$.

By Rothschild-Stein's "approximation theorem"(see [24, p. 273] or [1, Theorem 1.6]), one can locally approximate vector fields $\tilde{X}_{i}$ with left invariant vector fields $Y_{i}$ defined on a homogeneous group $G$ (which is actually $\mathbb{R}^{N}$ endowed with a suitable Lie group structure and allow the homogeneous dimension $Q$ ). This approximation is expressed by

$$
\tilde{X}_{i}\left(f\left(\Theta_{\xi}(\cdot)\right)\right)(\eta)=\left(Y_{i} f+R_{i}^{\xi} f\right)\left(\Theta_{\xi}(\eta)\right), \quad f \in C_{0}^{\infty}(G),
$$

where $\Theta_{\xi}(\eta)=\Theta(\eta, \xi)$ is a local diffeomorphism in $\mathbb{R}^{N}$, and $R_{i}^{\xi}$ is a vector field of local degree $\leq 0$ depending smoothly on $\xi$. More generally, for every multiple index $\alpha$ we have

$$
\tilde{X}_{\alpha}=Y_{\alpha}+R_{\alpha}^{\xi}
$$

with $R_{\alpha}^{\xi}$ a vector field of local degree $\leq|\alpha|-1$ depending smoothly on $\xi$ and $Y_{\alpha}$ a left invariant vector field of homogeneous degree $|\alpha|$ on the group $G$.

In the sequel we consider $G^{\prime}=\mathbb{R} \times G$ as a homogeneous group, with translations

$$
(t, \xi) \circ(s, \eta)=(t+s, \xi \circ \eta)
$$

dilations

$$
\delta(\lambda)(t, \xi)=\left(\lambda^{2} t, \delta(\lambda) \xi\right),
$$

and the homogeneous dimension $Q^{\prime}=Q+2$. Moreover, we will employ the quasidistance

$$
\tilde{d}(\xi, \eta)=\|\Theta(\xi, \eta)\|,
$$

introduced in [24], where $\|\cdot\|$ is the homogeneous norm in $G$. Note that the unit sphere $\{u \in G:\|u\|=1\}$ in $G$ coincides with the unit sphere $\Sigma_{N}$ in the Euclidean space $\mathbb{R}^{N}$. In order to make the unit sphere in $G^{\prime}$ coincide with $\Sigma_{N+1}$ in $\mathbb{R}^{N+1}$, we define a homogeneous norm of $G^{\prime}$ as follows

$$
\|(t, u)\|^{\prime}=\frac{1}{\sqrt{2}} \sqrt{\|u\|^{2}+\sqrt{\|u\|^{4}+4 t^{2}}}
$$


which satisfies

$$
\|(t, u)\|^{\prime}=\rho^{\prime} \Leftrightarrow\left|D\left(\left(\rho^{\prime}\right)^{-1}\right)(t, u)\right|=1 .
$$

Then the quasidistance on $G^{\prime}$ induced by $\|\cdot\|^{\prime}$ is

$$
\tilde{d}^{\prime}((t, \xi),(s, \eta))=\left\|(s, \eta)^{-1} \circ(t, \xi)\right\|^{\prime}=\frac{1}{\sqrt{2}} \sqrt{\tilde{d}(\xi, \eta)^{2}+\sqrt{\tilde{d}(\xi, \eta)^{4}+4|t-s|^{2}}}
$$

\section{Parabolic C-C distance.}

The constants in the final Morrey estimates in Theorem 1.1 will depend on the coefficients $a_{i j}(x)$ via "ellipticity constant" $\mu$ and the VMO modules of "lifted coefficients" $\tilde{a}_{i j}(t, x, h)=a_{i j}(t, x)$. These "lifted coefficients" are connected with the structure of homogeneous groups in the lifted space $\mathbb{R}^{N+1}$. Our ultimate target is to study functions and differential operators defined on $\mathbb{R}^{n+1}$. This requires us to express the $V M O$ modules of coefficients according to the structure induced by the original fields $X_{i}$ in $\mathbb{R}^{n}$. It is done from statements of [23] and [25]. We now collect some useful information.

For the system $X=\left(X_{1}, X_{2}, \cdots X_{q}\right)$ of Hörmander's vector fields, one can introduce a subelliptic metric $d_{X}($ see[1], [17], [25]):

DeFinition 5.1. Let $\Omega$ be a bounded domain in $\mathbb{R}^{n}$. An absolutely continuous curve $\gamma:[0, T] \rightarrow \Omega$ is called a subunit curve with respect to the system $X$, if for any $\xi \in \mathbb{R}^{n}$,

$$
\left\langle\gamma^{\prime}(t), \xi\right\rangle^{2} \leq \sum_{j=1}^{q}\left\langle X_{j}(\gamma(t)), \xi\right\rangle
$$

for a.e. $t \in[0, T]$. For any $x_{1}, x_{2} \in \Omega$, we define $d_{X}\left(x_{1}, x_{2}\right)=\inf \left\{T: \exists\right.$ a subunit curve $\left.\gamma:[0, T] \rightarrow \Omega, \gamma(0)=x_{1}, \gamma(T)=x_{2}\right\}$.

It is known (see [23]) that $d_{X}$ is a Carnot-Caratheodory distance, or briefly C-C distance. Let us define a parabolic Carnot-Caratheodory distance $d_{X}^{\prime}$ corresponding to $d_{X}$, namely,

$$
d_{X}^{\prime}((t, x),(s, y))=\frac{1}{\sqrt{2}} \sqrt{d_{X}(\xi, \eta)^{2}+\sqrt{d_{X}(\xi, \eta)^{4}+4|t-s|^{2}}}
$$


in the cylinder $\mathbb{R} \times \Omega$. Of course, the distance $d_{X}^{\prime}$ here is different from that in [2], but they are equivalent. Note that the doubling condition in the ball $\left(B_{r}\left(x_{0}\right), d_{X}, d x\right)$ holds (see [23]), and so does in the ball $\left(B_{r}\left(t_{0}, x_{0}\right), d_{X}^{\prime}, d t d x\right)$ (see [2]). Therefore we can apply the theories developed in Section 2, 3 to the space $\left(B_{r}\left(t_{0}, x_{0}\right), d_{X}^{\prime}, d t d x\right)$, and establish the relation between the balls in $\mathbb{R}^{n+1}$ induced by $\left\{X_{i}\right\}$ and that in $\mathbb{R}^{N+1}$ induced by $\left\{\tilde{X}_{i}\right\}$.

The C-C distance $d_{\tilde{X}}$ induced by the system $\tilde{X}=\left(\tilde{X}_{1}, \cdots, \tilde{X}_{q}\right)$ is equivalent to the quasidistance $\tilde{d}$ in (4.2), namely,

$$
c \tilde{d}(\xi, \eta) \leq d_{\tilde{X}}(\xi, \eta) \leq C \tilde{d}(\xi, \eta),
$$

for every $\xi, \eta \in \tilde{\Omega}$, where $\tilde{\Omega}$ is a bounded domain in $\mathbb{R}^{N}$. See Sanchez-Calle [25, Lemma 7, p. 153]. A similar statement is

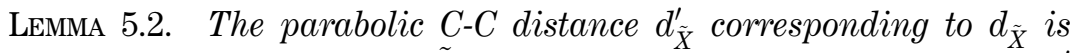
equivalent to the quasidistance $\tilde{d}^{\prime}$ induced by the homogeneous norm $\|\cdot\|^{\prime}$ on $G^{\prime}$, that is,

$$
c \tilde{d}^{\prime}((t, \xi),(s, \eta)) \leq d_{\tilde{X}}^{\prime}((t, \xi),(s, \eta)) \leq C \tilde{d}^{\prime}((t, \xi),(s, \eta)),
$$

for every $(t, \xi),(s, \eta) \in \tilde{U}$, where $\tilde{U}$ is a bounded domain in $\mathbb{R}^{N+1}$.

Sanchez-Calle in [25] pointed out that the volume of a ball for $d_{\tilde{X}}$ is essentially the volume of the ball of the same radius for $d_{X}$, times the volume of some ball in a $N-n$ dimensional Euclidean space, that is, there exist constants $r_{0}, c, C>0$ such that for every $z \in B_{X}(x, r)$ and $r \leq c r_{0}$,

$$
\begin{aligned}
& \frac{1}{C} \operatorname{vol}\left(B_{\tilde{X}}((x, h), r)\right) \\
\leq & \operatorname{vol}\left(B_{X}(x, r)\right) \cdot \operatorname{vol}\left\{h^{\prime} \in \mathbb{R}^{N-n}:\left(z, h^{\prime}\right) \in B_{\tilde{X}}((x, h), r)\right\} \\
\leq & C \operatorname{vol}\left(B_{\tilde{X}}((x, h), r)\right) .
\end{aligned}
$$

Here vol stands for the Lebesgue measure, $x$ is a point in $\mathbb{R}^{n}$ and $h$ a point in $\mathrm{R}^{N-n}$. Moreover,

$$
d_{\tilde{X}}\left((x, h),\left(x^{\prime}, h^{\prime}\right)\right) \geq d_{X}\left(x, x^{\prime}\right) .
$$

For the ball $B_{\tilde{X}}((t, x, h), r)$ and the parabolic C-C distance $d_{\tilde{X}}^{\prime}$, we have

Lemma 5.3. Let $\tilde{X}=\left(\tilde{X}_{1}, \cdots, \tilde{X}_{q}\right)$ be the lifted system in $\mathbb{R}^{N}$ for the system $X$ in $\mathbb{R}^{n}$. Then there exist constants $r_{0}, c, C>0$ such that for every 
$(s, z) \in B_{X}((t, x), r)$ and $r \leq c r_{0}$,

$$
\begin{aligned}
& \frac{1}{C} \operatorname{vol}\left(B_{\tilde{X}}((t, x, h), r)\right) \\
\leq & \operatorname{vol}\left(B_{X}((t, x), r)\right) \cdot \operatorname{vol}\left\{h^{\prime} \in \mathbb{R}^{N-n}:\left(s, z, h^{\prime}\right) \in B_{\tilde{X}}((t, x, h), r)\right\} \\
\leq & C \operatorname{vol}\left(B_{\tilde{X}}((t, x, h), r)\right) .
\end{aligned}
$$

Here $(t, x)$ denotes a point in $\mathbb{R}^{n+1}$ and $h$ a point in $\mathbb{R}^{N-n}$. Moreover,

$$
d_{\tilde{X}}^{\prime}\left((t, x, h),\left(t^{\prime}, x^{\prime}, h^{\prime}\right)\right) \geq d_{X}^{\prime}\left((t, x),\left(t^{\prime}, x^{\prime}\right)\right) .
$$

Proof. Using Lemma 3.6 in [2] shows that $\operatorname{vol}\left(B_{X}((t, x), r)\right)$ is equivalent to $r^{2} \operatorname{vol}\left(B_{X}(x, r)\right)$, and $\operatorname{vol}\left(B_{\tilde{X}}((t, x, h), r)\right)$ is equivalent to $r^{2} \operatorname{vol}\left(B_{\tilde{X}}((x, h), r)\right)$. Therefore, it is easy to get (5.4) from (5.2). Moreover, (5.5) is simply obtained from (5.1) and (5.3).

We are now ready to prove the following result:

THEOREM 5.4. The function $f(t, x)$ belongs to the space BMO (or VMO) on the homogeneous space $\left(S, d_{X}^{\prime}, d t d x\right)$ if and only if the function $\tilde{f}(t, \xi)=\tilde{f}(t, x, h)=f(t, x)$ is in the space BMO (or VMO) on the homogeneous space $\left(\tilde{S}, \tilde{d}^{\prime}, d t d \xi\right)$.

Proof. We follow the method in [1]. By John-Nirenberg lemma on homogeneous spaces (see [6]) and (5.4),

$$
\begin{aligned}
& \operatorname{vol}\left(B_{\tilde{X}}((t, x, h), r)\right)^{-1} \int_{B_{\tilde{X}}((t, x, h), r)}\left|\tilde{f}\left(s, y, h^{\prime}\right)-\tilde{f}_{B_{\tilde{X}}((t, x, h), r)}\right| d s d y d h^{\prime} \\
& \leq \operatorname{vol}\left(B_{\tilde{X}}((t, x, h), r)\right)^{-1 / 2}\left(\int_{\left.B_{\tilde{X}}(t, x, h), r\right)}\left|\tilde{f}\left(s, y, h^{\prime}\right)-c\right|^{2} d s d y d h^{\prime}\right)^{1 / 2} \\
& \leq \operatorname{vol}\left(B_{\tilde{X}}((t, x, h), r)\right)^{-1 / 2}\left(\int _ { B _ { X } ( ( t , x ) , r ) } \operatorname { v o l } \left\{h^{\prime}:\left(s, y, h^{\prime}\right)\right.\right. \\
& \left.\left.\in B_{\tilde{X}}((t, x, h), r)\right\}|f(s, y)-c|^{2} d s d y\right)^{1 / 2}
\end{aligned}
$$

(by (5.4), we assume $r \leq c r_{0}^{2}$ ) 


$$
\begin{aligned}
& \leq C \operatorname{vol}\left(B_{X}((t, x), r)\right)^{-1 / 2}\left(\int_{B_{X}((t, x), r)}|f(s, y)-c|^{2} d s d y\right)^{1 / 2} \\
& \leq C \frac{1}{\left(B_{X}((t, x), r)\right)} \int_{B_{X}((t, x), r)}\left|f(s, y)-f_{B_{X}((t, x), r)}\right| d y,
\end{aligned}
$$

with $c=f_{B_{X}}((t, x), r)$.

This implies that if $f(t, x)$ belongs to $B M O$ (or $V M O)$ on $\left(S, d_{X}^{\prime}, d t d x\right)$, then $\tilde{f}(t, \xi)$ belongs to $B M O$ (or $V M O)$ on $\left(\tilde{S}, d_{\tilde{X}}^{\prime}, d t d \xi\right)$. The converse can be proved analogously. Finally, by Lemma 5.2 and the doubling property, we see that the spaces $B M O$ (or $V M O$ ) defined on $\left(\tilde{S}, d_{\tilde{X}}^{\prime}, d t d \xi\right)$ and $\left(\tilde{S}, \tilde{d}^{\prime}, d t d \xi\right)$ coincide.

\section{Differential operators and fundamental solutions.}

We now define several differential operators that we will handle in the subsequence. The main objective to study is the operator

$$
H=\partial_{t}-\sum_{i, j=1}^{q} a_{i j}(t, x) X_{i} X_{j}
$$

where $X_{i}$ 's in $\Omega$ satisfy (H1) and the matrix $\left\{a_{i j}(x)\right\}$ satisfies (H2)-(H3) in Section 1. For $(t, \xi)=(t, x, h) \in \tilde{U} \subset \mathbb{R} \times \tilde{\Omega}$, let $\tilde{a}_{i j}(t, x, h)=a_{i j}(t, x)$ and

$$
\tilde{H}=\partial_{t}-\sum_{i, j=1}^{q} \tilde{a}_{i j}(t, \xi) \tilde{X}_{i} \tilde{X}_{j}
$$

be the lifted operator defined in $\tilde{\Omega}=\Omega \times \mathbb{R}^{N-n}$. Now, we freeze $\tilde{H}$ at some point $\left(t_{0}, \xi_{0}\right) \in \tilde{U}$ and obtain a frozen lifted operator:

$$
\tilde{H}_{0}=\partial_{t}-\sum_{i, j=1}^{q} \tilde{a}_{i j}\left(t_{0}, \xi_{0}\right) \tilde{X}_{i} \tilde{X}_{j} .
$$

To study $\tilde{H}_{0}$, we first discuss its approximating operator

$$
H_{0}=\partial_{t}-\sum_{i, j=1}^{q} \tilde{a}_{i j}\left(t_{0}, \xi_{0}\right) Y_{i} Y_{j},
$$

defined in the group $\mathbb{R} \times G$. It is seen from [3] that for every $\left(t_{0}, \xi_{0}\right) \in \tilde{U}$, the operator $H_{0}$ is hypoelliptic and homogeneous of degree 2 in $\mathbb{R} \times G$; Moreover, the transpose $H_{0}^{T}$ of $H_{0}$ is also hypoelliptic. 
Applying the results proved by Folland (see Proposition 1.8, Theorem 2.1 and Corollary 2.8 in [15]) to $H_{0}$, we know that $H_{0}$ has a fundamental solution of homogeneous of order $2-Q^{\prime}$, denoted by $\Gamma\left(t_{0}, \xi_{0} ; \cdot, \cdot\right)$. It is dependent of $\tilde{a}_{i j}\left(t_{0}, \xi_{0}\right)$. Moreover, for $i, j=1, \cdots q$, denote

$$
\Gamma_{i j}\left(t_{0}, \xi_{0} ; t, u\right)=Y_{i} Y_{j}\left[\Gamma\left(t_{0}, \xi_{0} ; t, \cdot\right)\right](u) .
$$

The following summarizes the properties of $\Gamma\left(t_{0}, \xi_{0} ; \cdot, \cdot\right)$ and $\Gamma_{i j}\left(t_{0}, \xi_{0} ; t, u\right)$ that will be used later (see [1]).

Lemma 6.1. For every $\left(t_{0}, \xi_{0}\right) \in \mathbb{R}^{N+1}$, one has

(a) $\Gamma\left(t_{0}, \xi_{0} ; \cdot, \cdot\right), \Gamma_{i j}\left(t_{0}, \xi_{0} ; \cdot\right) \in C^{\infty}\left(\mathbb{R}^{N+1} \backslash\{0\}\right)$;

(b) $\Gamma\left(t_{0}, \xi_{0} ; \cdot, \cdot\right)$ is homogeneous of degree $2-Q^{\prime}$ and $\Gamma_{i j}\left(t_{0}, \xi_{0} ; \cdot, \cdot\right)$ is homogeneous of degree $-Q^{\prime}$;

(c) For every test function $f$ and every $(s, v) \in \mathbb{R}^{N+1}$,

$f(s, v)=\left(H_{0} f * \Gamma\left(t_{0}, \xi_{0} ; \cdot, \cdot\right)\right)(s, v)=\int_{\mathbb{R}^{N+1}} \Gamma\left(t_{0}, \xi_{0} ; s-t, u^{-1} \circ v\right) H_{0} f(t, u) d t d u ;$

furthermore, for every $i, j=1,2, \cdots q$, there exists uniformly bounded constants $\alpha_{i j}\left(t_{0}, \xi_{0}\right)$ such that

$$
\begin{aligned}
Y_{i} Y_{j} f(s, v)=P . V . \int_{\mathbb{R}^{N+1}} \Gamma_{i j}\left(t_{0}, \xi_{0} ; s-t, u^{-1} \circ v\right) & H_{0} f(t, u) d t d u \\
& +\alpha_{i j}\left(t_{0}, \xi_{0}\right) H_{0} f(s, v)
\end{aligned}
$$

(d) For any $R>r>0$,

$$
\int_{r<\|(t, u)\|^{\prime}<R} \Gamma_{i j}\left(t_{0}, \xi_{0} ; t, u\right) d t d u=\int_{\|(t, u)\|^{\prime}=1} \Gamma_{i j}\left(t_{0}, \xi_{0} ; t, u\right) d \sigma(t, u)=0 ;
$$

(e) For any multiple index $\beta$, there exists a constant $c_{1}$ such that for any $i, j=1, \cdots q$,

$$
\sup _{\|(s, u)\|^{\prime}=1,(t, \xi) \in \mathbb{R}^{N+1}}\left|\left(\frac{\partial}{\partial u}\right)^{\beta} \Gamma_{i j}(t, \xi ; s, u)\right| \leq c_{1} .
$$

We will define the kernels of the operator $\tilde{H}_{0}$ in (6.3), which induce singular and fractional integrals on the homogeneous space. Here we follow the way in Rothschild-Stein [24] and Bramanti-Brandolini [1-2].

Definition 6.2. Let $\Gamma\left(t_{0}, \xi_{0} ; \cdot, \cdot\right)$ be the fundamental solution of $H_{0}$. We say that $k\left(t_{0}, \xi_{0} ; t, \xi, \eta\right)$ is a frozen kernel of type $l$, for some nonnegative 
integer $l$, if for every positive integer $m$,

$$
\begin{aligned}
k\left(t_{0}, \xi_{0} ; t, \xi, \eta\right)= & \sum_{i=1}^{H_{m}} a_{i}(\xi) b_{i}(\eta)\left[D_{i} \Gamma\left(t_{0}, \xi_{0} ; \cdot, \cdot\right)\right](t, \Theta(\eta, \xi)) \\
& +a_{0}(\xi) b_{0}(\eta)\left[D_{0} \Gamma\left(t_{0}, \xi_{0} ; \cdot \cdot \cdot\right)\right](t, \Theta(\eta, \xi)),
\end{aligned}
$$

where $a_{i}, b_{i}\left(i=0,1, \cdots, H_{m}\right)$ are test functions, $D_{i}$ are differential operators such that: for $i=1, \cdots, H_{m}, D_{i}$ is homogeneous of degree $\leq 2-l$ (so that $D_{i} \Gamma\left(t_{0}, \xi_{0} ; \cdot\right)$ is a homogeneous function of degree $\left.\geq l-Q\right)$, and $D_{0}$ is a differential operator such that $D_{0} \Gamma\left(t_{0}, \xi_{0} ; \cdot\right)$ has $m$-th derivatives with respect to the vector fields $Y_{i}(i=1, \cdots, q)$.

We say that $T\left(t_{0}, \xi_{0}\right)$ is a frozen operator of type $l \geq 1$ if $k\left(t_{0}, \xi_{0} ; t, \xi, \eta\right)$ is a frozen kernel of type $l$ and

$$
T\left(t_{0}, \xi_{0}\right) f(t, \xi)=\int_{-\infty}^{t} \int_{\mathbb{R}^{N}} k\left(t_{0}, \xi_{0} ; t-s, \xi, \eta\right) f(s, \eta) d s d \eta
$$

we say that $T\left(t_{0}, \xi_{0}\right)$ is a frozen operator of type 0 (or "frozen singular integral") if $k\left(t_{0}, \xi_{0} ; t, \xi, \eta\right)$ is frozen kernel of type 0 and

$$
T\left(t_{0}, \xi_{0}\right) f(t, \xi)=P V \int_{-\infty}^{t} \int_{\mathbb{R}^{N}} k\left(t_{0}, \xi_{0} ; t-s, \xi, \eta\right) f(s, \eta) d s d \eta+\alpha\left(t_{0}, \xi_{0}, t, \xi\right) f(t, \xi),
$$

where the function $\alpha$ is bounded.

If $k\left(t_{0}, \xi_{0} ; t, \xi, \eta\right)$ is a frozen kernel of type $l$, then we say that $k(t, \xi ; t, \xi, \eta)$ is a variant kernel of type $l$, and

$$
T(t, \xi) f(t, \xi)=\int_{-\infty}^{t} \int_{\mathbb{R}^{N}} k(t, \xi ; t-s, \xi, \eta) f(s, \eta) d s d \eta
$$

is a variant operator of type $l$ (if $l=0$, the integral is taken in principal value sense and the term $\alpha(t, \xi, t, \xi) f(t, \xi)$ is added).

The following two Lemmas were proved in [1, Lemma 2.8] and [24, Theorem 8], respectivly.

Lemma 6.3. If $k\left(t_{0}, \xi_{0} ; t, \xi, \eta\right)$ is a frozen kernel of type $l \geq 1$, then $\left(\tilde{X}_{i} k\right)\left(t_{0}, \xi_{0} ; t, \cdot, \eta\right)(\xi)$ is a frozen kernel of type $l-1$. 
Lemma 6.4. If $T\left(t_{0}, \xi_{0}\right)$ is a frozen operator of type of $l \geq 1$, then $\tilde{X}_{i} T\left(t_{0}, \xi_{0}\right)$ is a frozen operator of type $l-1$, and

$$
\tilde{X}_{i} T\left(t_{0}, \xi_{0}\right)=\sum_{k=1}^{q} T_{k}\left(t_{0}, \xi_{0}\right) \tilde{X}_{k}+T_{0}\left(t_{0}, \xi_{0}\right)
$$

for suitable frozen operator $T_{k}\left(t_{0}, \xi_{0}\right)(k=0,1, \cdots q)$ of type $l$.

We will use some properties of cutoff functions.

Lemma 6.5. For any $0<\rho<r$ and $(t, \xi) \in \mathbb{R}^{N+1}$, there exists $\varphi \in C_{0}^{\infty}\left(\mathbb{R}^{N+1}\right)$ such that

(i) $0 \leq \varphi \leq 1, \varphi \equiv 1$ on $\tilde{B}_{\rho}(t, \xi)$ under the lifted distance $\tilde{d}^{\prime}$ and sprt $\varphi \subseteq \tilde{B}_{r}(t, \xi)$;

(ii) $\left|\partial_{t}^{h} D^{k} \varphi\right| \leq \frac{c_{k, h}}{(r-\rho)^{k+2 h}}$, for $k, h \in \mathrm{N}$;

Its proof is similar to the proof of Lemma 6.2 in [2], just with slight and clear changes.

Let $D^{k}=\tilde{X}_{i_{1}} \cdots \tilde{X}_{i_{k}}, 1 \leq i_{j} \leq q, 1 \leq j \leq k$, and write

$$
\tilde{B}_{\rho}(t, \xi) \prec \varphi \prec \tilde{B}_{r}(t, \xi)
$$

for the function $\varphi$ satisfying Lemma 6.5.

Next we establish the Morrey estimates of the variable operator of type 0 by using results on homogeneous spaces in Section 3. The main result in this section is the following.

THeOREm 6.6. Let $T$ be a variable operator of type 0 and $\tilde{U} \subset \mathbb{R}^{N+1}$. Then for every $p \in(1, \infty)$ and every $\lambda \in\left(0, Q^{\prime}\right)$, there exists $c=c(p, \lambda, T)$ such that $a \in B M O(\tilde{U})$

(i) $\|T f\|_{p, \lambda ; \tilde{U}} \leq c\|f\|_{p, \lambda ; \tilde{U}}$, for every $f \in L^{p, \lambda}(\tilde{U})$;

(ii) $\|T(a f)-a \cdot T f\|_{p, \lambda ; \tilde{U}} \leq c\|a\|_{*}\|f\|_{p, \lambda ; \tilde{U}}$, for every $f \in L^{p, \lambda}(\tilde{U})$,

(iii) Moreover, for every $a \in V M O$ and every $\varepsilon>0$, there exists $r=r\left(p, \lambda, T, \eta_{a}, \varepsilon\right)>0$ such that for every $\xi_{0} \in \mathbb{R}^{N}$ and every $f \in L^{p, \lambda}$ with sprt $f \subseteq \tilde{B}_{r}\left(t_{0}, \xi_{0}\right)$,

$$
\|T(a f)-a \cdot T f\|_{p, \lambda ; \tilde{B}_{r}} \leq \varepsilon\|f\|_{p, \lambda ; \tilde{B}_{r}} .
$$

(Recall that $\eta_{a}$ denotes the VMO moduli of a, see Definition 2.2.) 
The way of proving Theorem 6.6 is inspired by the method in [1]. It is worthy to note that (iii) follows from (ii) by using a localization technique which was first employed in [8]. So we only need to prove (i)-(ii).

In order to complete the proof of Theorem 6.6, we apply CalderónZygmund's technique of expansion in spherical harmonics (see [8]) and the estimate of constant kernels (Proposition 6.8 below). Let $\left\{Y_{k m}\right\}_{\substack{m=0,1,2, \ldots \\ k=1, \ldots, g_{m}}}$ be a complete orthonormal system of spherical harmonics in $L^{2}\left(\Sigma_{N+1}\right)$, where $m$ is the degree of the polynomial and $g_{m}$ is the dimension of the space of spherical harmonics of degree $m$ in $\mathbb{R}^{N+1}$. Then

$$
\left|\left(\frac{\partial}{\partial \eta}\right)^{\beta} Y_{k m}(\eta)\right| \leq C(N) m^{(N-1) / 2+|\beta|} \text {, for } \eta \in \Sigma_{N+1} .
$$

It is known that

$$
g_{m} \leq C(N) m^{N-1}, \text { for } m=1,2, \cdots .
$$

For any fixed $z=(t, \xi) \in \mathbb{R}^{N+1}, \eta \in \Sigma_{N+1}$, we can write the expansion:

$$
\Gamma(z ; \eta)=\sum_{m=0}^{\infty} \sum_{k=1}^{g_{m}} c^{k m}(z) Y_{k m}(\eta) .
$$

For $\eta \in \mathbb{R}^{N+1}$, let $\eta^{\prime}=\delta\left(\|\eta\|^{-1}\right) \eta$; recall that $\eta^{\prime} \in \Sigma_{N+1}$ from Section 4 . By the homogeneity of $\Gamma$, we have

$$
\Gamma(z ; \eta)=\sum_{m=0}^{\infty} \sum_{k=1}^{g_{m}} c^{k m}(z) \frac{Y_{k m}\left(\eta^{\prime}\right)}{\|\eta\|^{Q^{\prime}-2}} .
$$

Coefficients $c^{k m}$ in above formula have the bound (see [3]): for every positive integer $l$, there exists a constant $C=C(l, \mu, N)$ such that

$$
\sup _{z \in \mathbb{R}^{N+1}}\left|c^{k m}(z)\right| \leq C m^{-2 l},
$$

for every $m=1,2, \cdots, k=1, \cdots, g_{m}$.

Now, for $z \in \mathbb{R}^{N+1}$ and $z^{\prime} \in \Sigma_{N+1}$, let

$$
H_{k m}(z)=\frac{Y_{k m}\left(z^{\prime}\right)}{\|z\|^{Q^{\prime}-2}} .
$$

Definition 6.7. We say that $K_{k m}(t, \xi, s, \eta)=a(\xi)\left[D H_{k m}(\cdot)\right](t-s$, $\Theta(\eta, \xi)) b(\eta)$ is a constant kernel of type $l$, if the differential operator $D$ is homogeneous of degree $2-l$, so that $D H_{k m}$ is homogeneous of degree $l-Q^{\prime}$. 
The following proposition gives properties of the constant kernel of type $l$.

Proposition 6.8. Let $W$ be a function defined on $G^{\prime}$, smooth outside the origin and homogeneous of degree $l-Q^{\prime}$, and let

$$
K(t, \xi, s, \eta)=a(\xi) W(t-s, \Theta(\eta, \xi)) b(\eta),
$$

where $a(\xi)$ and $b(\eta)$ are fixed test functions. Then

such that

(i) $K$ satisfies the growth condition: there exists a constant $c>0$

$$
|K(t, \xi, s, \eta)| \leq c \cdot \sup _{\Sigma_{N+1}}|W| \cdot \tilde{d}^{\prime}(t, \xi, s, \eta)^{l-Q^{\prime}} ;
$$

(ii) $K$ satisfies the mean value inequality: there exist constants $c>0, M>1$ such that for every $\xi_{0}, \xi, \eta$,

$$
\left|K\left(t_{0}, \xi_{0}, s, \eta\right)-K(t, \xi, s, \eta)\right|+\left|K\left(s, \eta, t_{0}, \xi_{0}\right)-K(s, \eta, t, \xi)\right|
$$

$$
\leq c \cdot \sup _{\Sigma_{N+1}}|W| \cdot \frac{\tilde{d}^{\prime}\left(t_{0}, \xi_{0}, t, \xi\right)}{\tilde{d}^{\prime}\left(t_{0}, \xi_{0}, s, \eta\right)^{Q^{\prime}-l+1}},
$$

with $\tilde{d}^{\prime}\left(t_{0}, \xi_{0}, s, \eta\right) \geq M \tilde{d}^{\prime}\left(t_{0}, \xi_{0}, t, \xi\right)$;

(iii) If $l=0$ and $W$ satisfies the vanishing property:

$$
\int_{r<\|(t, u)\|^{\prime}<R} W(t, u) d t d u=0, \text { for every } R>r>0,
$$

then $K$ satisfies the cancellation property:

$$
\left|\int_{r<\tilde{d}^{\prime}(t, \xi, s, \eta)<R} K(t, \xi, s, \eta) d s d \eta\right| \leq c \cdot \sup _{\Sigma_{N+1}}|W| \cdot(R-r), \text { for every } R>r>0 .
$$

All constants in (i)-(ii)-(iii) are independent of W.

REMark 6.9. The estimate in (ii) is sharper than those in Proposition 2.17 of [1] and Proposition 6.4 of [2].

Proof of Proposition 6.8. (i) The claim is trivial, by the homogeneity of $W$. 
(ii) With the similar proof to Proposition 2.17 in [2], fix $\left(t_{0}, \xi_{0}\right),(s, \eta)$, and let $M r=\tilde{d}^{\prime}\left(t_{1}, \xi_{1}, s, \eta\right)$. Then the condition $\tilde{d}^{\prime}\left(t_{0}, \xi_{0}, s, \eta\right)>M \tilde{d}^{\prime}\left(t_{0}, \xi_{0}, t, \xi\right)$ means that $(t, \xi)$ is a point in $\tilde{B}_{r}\left(t_{0}, \xi_{0}\right)$. From Lemma 6.5 , we take a cutoff function $\varphi(t, \xi)$ such that

$$
\tilde{B}_{r}\left(t_{0}, \xi_{0}\right) \prec \varphi \prec \tilde{B}_{M^{\prime} r}\left(t_{0}, \xi_{0}\right), \quad 1<M^{\prime}<M,
$$

and let

$$
f(t, \xi)=K(t, \xi, s, \eta) \varphi(t, \xi) .
$$

Then using Proposition 4.2 in [2] obtains

$$
\left|K\left(t_{0}, \xi_{0}, s, \eta\right)-K(t, \xi, s, \eta)\right|=\left|f\left(t_{0}, \xi_{0}\right)-f(t, \xi)\right|
$$

$$
\leq \tilde{d}^{\prime}\left(t_{0}, \xi_{0}, t, \xi\right)\left\{\sup _{(\tau, \zeta) \in \tilde{B}_{M^{\prime} r}\left(t_{0}, \xi_{0}\right)}|\tilde{X} f(\tau, \zeta)|+M^{\prime} r \sup _{(\tau, \zeta) \in \tilde{B}_{M^{\prime} r}\left(t_{0}, \xi_{0}\right)} f_{t}(\tau, \zeta)\right\} .
$$

Note

$$
\tilde{X}_{j} f(\tau, \zeta)=\tilde{X}_{j} K(\tau, \zeta, s, \eta) \varphi(\tau, \zeta)+K(\tau, \zeta, s, \eta) \tilde{X}_{j} \varphi(\tau, \zeta):=\mathrm{I}+\mathrm{II} .
$$

By Lemma $6.3, \tilde{X}_{j} K(\tau, \cdot, s, \eta)(\zeta)$ is homogeneous of type $l-Q^{\prime}-1 \leq 0$, so

$$
\begin{aligned}
|\mathrm{I}| & \leq c \cdot \sup _{\Sigma_{N+1}}|W| \cdot \tilde{d}^{\prime}(\tau, \zeta, s, \eta)^{l-Q^{\prime}-1} \varphi(\tau, \zeta) \\
& \leq c \cdot \sup _{\Sigma_{N+1}}|W| \cdot r^{l-Q^{\prime}-1} \leq c \cdot \sup _{\Sigma_{N+1}}|W| \cdot \tilde{d}^{\prime}\left(t_{0}, \xi_{0}, s, \eta\right)^{l-Q^{\prime}-1}, \\
|\mathrm{II}| & \leq c \cdot \sup _{\Sigma_{N+1}}|W| \cdot \tilde{d}^{\prime}(\tau, \zeta, s, \eta)^{l-Q^{\prime}} \varphi(\tau, \zeta) \cdot \frac{c}{r} \\
& \leq c \cdot \sup _{\Sigma_{N+1}}|W| \cdot r^{l-Q^{\prime}-1} \leq c \cdot \sup _{\Sigma_{N+1}}|W| \cdot \tilde{d}^{\prime}\left(t_{0}, \xi_{0}, s, \eta\right)^{l-Q^{\prime}-1},
\end{aligned}
$$

where we have used $\tilde{d}^{\prime}(\tau, \zeta, s, \eta) \geq c r$ for $(\tau, \zeta) \in \tilde{B}_{M^{\prime} r}\left(t_{0}, \xi_{0}\right)$ and $2 r=\tilde{d}^{\prime}\left(t_{0}, \xi_{0}, s, \eta\right)$.

Since $\partial_{t} K(\cdot, \zeta, s, \eta)(\tau)$ is homogeneous of type $l-Q^{\prime}-2 \leq 0$, we have

$$
\left|f_{t}(\tau, \zeta)\right|=\left|\partial_{t} K(\cdot, \zeta, s, \eta)(\tau) \varphi(\tau, \zeta)\right|+\left|K(\tau, \zeta, s, \eta) \varphi_{t}(\tau, \zeta)\right| \leq c \cdot \sup _{\Sigma_{N+1}}|W| \cdot r^{l-Q^{\prime}-2} .
$$

Therefore,

$$
r \sup _{(\tau, \zeta) \in \tilde{B}_{M^{\prime} r}\left(t_{0}, \xi_{0}\right)}\left|f_{t}(\tau, \zeta)\right| \leq c \cdot \sup _{\Sigma_{N+1}}|W| \cdot r^{l-Q^{\prime}-1} \leq c \cdot \sup _{\Sigma_{N+1}}|W| \cdot \tilde{d}^{\prime}\left(t_{0}, \xi_{0}, s, \eta\right)^{l-Q^{\prime}-1} .
$$


From (6.13) one gets

$$
\left|K\left(t_{0}, \xi_{0}, s, \eta\right)-K(t, \xi, s, \eta)\right| \leq c \cdot \sup _{\Sigma_{N+1}}|W| \cdot \frac{\tilde{d}^{\prime}\left(t_{0}, \xi_{0}, t, \xi\right)}{\tilde{d}^{\prime}\left(t_{0}, \xi_{0}, s, \eta\right)^{Q^{\prime}-l+1}},
$$

for $\tilde{d}^{\prime}\left(t_{0}, \xi_{0}, s, \eta\right) \geq M \tilde{d}^{\prime}\left(t_{0}, \xi_{0}, t, \xi\right)$. Similarly,

$$
\left|K\left(s, \eta, t_{0}, \xi_{0}\right)-K(s, \eta, t, \xi)\right| \leq c \cdot \sup _{\Sigma_{N+1}}|W| \cdot \frac{\tilde{d}^{\prime}\left(t_{0}, \xi_{0}, t, \xi\right)}{\tilde{d}^{\prime}\left(t_{0}, \xi_{0}, s, \eta\right)^{Q^{\prime}-l+1}} .
$$

(iii) The claim can be proved as in the proof of Proposition 2.17 in [1], just adding a time variable.

Proof of Theorem 6.6. - The multiplicative part of the variable operator $T$, that is, $f(t, \xi) \mapsto \alpha(t, \xi, t, \xi) f(t, \xi)$, clearly satisfies the demanded estimates. By Definition 6.2, if $k(t, \xi ; t-s, \xi, \eta)$ is a variable kernel of type 0 , then we separate it into singular and regular parts:

$$
\begin{aligned}
& k(t, \xi ; t-s, \xi, \eta) \equiv S(t, \xi ; t-s, \xi, \eta)+R(t, \xi ; t-s, \xi, \eta) \\
& =\left.\left\{\sum_{i=1}^{H_{m}} a_{i}(\xi) b_{i}(\eta)\left[D_{i} \Gamma\left(t_{0}, \xi_{0} ; \cdot\right)\right](t-s, \Theta(\eta, \xi))\right\}\right|_{\left(t_{0}, \xi_{0}\right)=(t, \xi)} \\
& \quad+\left.\left\{a_{0}(\xi) b_{0}(\eta)\left[D_{0} \Gamma\left(t_{0}, \xi_{0} ; \cdot\right)\right](t-s, \Theta(\eta, \xi))\right\}\right|_{\left(t_{0}, \xi_{0}\right)=(t, \xi)}
\end{aligned}
$$

where $a_{i}, b_{i}\left(i=0,1, \cdots g_{m}\right)$ are test functions, $D_{i}\left(i=1, \cdots g_{m}\right)$ are differential operators homogeneous of degree $\leq 2$ (so that $D_{i} \Gamma\left(t_{0}, \xi_{0} ; \cdot\right)$ is a homogeneous function of degree $\geq-Q^{\prime}$ ), and $D_{0}$ is a differential operator such that $D_{0} \Gamma\left(t_{0}, \xi_{0} ; \cdot\right)$ is locally bounded. Here "singular" and "regular" just mean "unbounded" and "bounded", respectively.

For the regular part, using the same argument as Theorem 2.11 in [1], we easily illustrate that the operators corresponding to $R(t, \xi ; t-s, \xi, \eta)$ satisfy the conditions of Lemma 3.1, and then the conclusion of Theorem 6.6 holds for these operators.

Now, let us deal with the singular part. From (6.8)-(6.10) and a similar deduction in [1], we write

$$
\begin{aligned}
S(t, \xi ; t-s, \xi, \eta) & =\sum_{m=0}^{\infty} \sum_{k=1}^{g_{m}} \sum_{i=1}^{H_{m}} c^{k m}(t, \xi) a_{i}(\xi)\left[D_{i} H_{k m}(\cdot)\right](t-s, \Theta(\eta, \xi)) b_{i}(\eta) \\
& =\sum_{m=0}^{\infty} \sum_{k=1}^{g_{m}} c^{k m}(t, \xi) K_{k m}(t, \xi, s, \eta),
\end{aligned}
$$

where $D_{i}$ is a differential operator of homogeneous of type $\leq 2$, $K_{k m}(t, \xi, s, \eta)$ are constant kernels of type $\geq 0$. 
Our next step is to gain $L^{p, \lambda}$ estimates for the constant operators of type $l \geq 0$ :

$$
T_{k m} f(t, \xi)=\int_{\mathbb{R}^{N+1}} K_{k m}(t, \xi, s, \eta) f(s, \eta) d \eta d s,
$$

and their commutators with $B M O$ functions. Since $K_{k m}$ is a constant kernel of type $\geq 0$, by Proposition 6.8 (i),

$$
\begin{aligned}
\left|K_{k m}(t, \xi, s, \eta)\right| \leq c \tilde{d}^{\prime}(t, \xi, s, \eta)^{l-Q^{\prime}} \cdot \sup _{\Sigma_{N+1}} \mid & D H_{k m}(\cdot) \mid \\
& \leq c \tilde{d}^{\prime}(t, \xi, s, \eta)^{l-Q^{\prime}} \cdot m^{(N+1) / 2+1},
\end{aligned}
$$

with $D$ a differential operator of homogeneous of degree $2-l$, and

$$
\left|\left(\frac{\partial}{\partial u}\right)^{\beta} H_{k m}(u)\right| \leq c(\beta, N) m^{(N-1) / 2+|\beta|}, \text { for } u \in \Sigma_{N+1},
$$

(see Lemma 2.16 in [1]).

We investigate the cases $l>0$ and $l=0$, respectively.

If $l>0$, by (6.15) and Theorem 3.2, the operator $T_{k m}$ satisfies estimates like (3.6)-(3.7) with constant $c \cdot m^{(N+1) / 2+1}$, for $p \in\left(1, Q^{\prime} / l\right), \lambda \in\left(0, Q^{\prime}-p l\right)$, $1 / q=1 / p-l / Q^{\prime}$, and $\mu=\frac{\lambda}{p} q$. Hence, by the boundedness of the domain $\Omega$ and Lemma 3.3, $T_{k m}$ satisfies estimates (3.3)-(3.4) for $p \in\left(1, Q^{\prime} / l\right)$ and $\lambda \in\left(0, Q^{\prime}-p l\right)$. It is evident to see that the transpose of $T_{k m}$ also satisfies (3.3)-(3.4) for $p \in\left(1, Q^{\prime} / l\right)$ and $\lambda \in\left(0, Q^{\prime}-p l\right)$; therefore, by duality, $T_{k m}$ satisfies (3.3)-(3.4) for $p \in\left(Q^{\prime} /\left(Q^{\prime}-l\right), \infty\right)$ and $\lambda \in\left(0, Q^{\prime}\right)$. Finally, Theorem 3.9 and Corollary 3.10 show the boundedness in the full range $p \in(1, \infty)$ and $\lambda \in\left(0, Q^{\prime}\right)$.

If $l=0$, by $(6.16)$, the kernel $K_{k m}(\xi, \eta)$ satisfies mean value inequality (2.18) with constant $c \cdot m^{(N+1) / 2+2}$. Since $D H_{k m}$ fits the condition of Proposition 6.8 (iii) (see $[15,18]$ ), the function $D H_{k m}$ satisfies the cancellation property. Hence, the kernel $K_{k m}(\xi, \eta)$ satisfies the vanishing property of (6.12) with constant $c \cdot m^{(N+1) / 2+1}$. Using Theorem 2.12 in [1] or Theorem 3.1 in [4] implies that $T_{k m}$ has the $L^{p}$ estimates like (3.3)-(3.4) for $p \in(1, \infty)$ with constant $c \cdot m^{(N+1) / 2+2}$. Then we conclude that $T_{k m}$ satisfies estimates (3.3)-(3.4) for $p \in(1, \infty)$ and $\lambda \in\left(0, Q^{\prime}\right)$.

Recall the estimate $(6.9)$ on the coefficients $c^{k m}(t, \xi)$ in (6.14) and the inequality (6.7). Putting these facts together with the above arguments, we get estimates (3.3)-(3.4) for the operator with kernel $S(t, \xi ; t, \xi, s, \eta)$ for every $p \in(1, \infty)$ and $\lambda \in\left(0, Q^{\prime}\right)$. This ends the proof of Theorem 6.6. 


\section{Local Morrey estimates in $S_{X}^{2, p, \lambda}(U)$.}

In this Section we will use several kinds of Sobolev-Morrey spaces:

DEFINITION 7.1. Let $Z=\left(Z_{1}, Z_{2}, \cdots Z_{q}\right)$ be any system of smooth vector fields in $\mathbb{R}^{n}$. We define Sobolev-Morrey spaces $S_{Z}^{k, p, \lambda}\left(\mathbb{R}^{n+1}\right)$ of $L^{p, \lambda}$ functions with $k$-th derivatives with respect to vector fields $Z_{i}$ 's, in $L^{p, \lambda}\left(1<p<\infty, 0<\lambda<q^{\prime}\right)$. The Sobolev-Morrey norm is defined by

$$
\begin{aligned}
\|f\|_{S_{Z}^{k, p, \lambda}\left(\mathbb{R}^{n+1}\right)}= & \|f\|_{L^{p, \lambda}\left(\mathbb{R}^{n+1}\right)} \\
& +\sum_{h+2 m \leq k}\left(\sum_{j_{i}=1}^{q}\left\|Z_{j_{1}} Z_{j_{2}} \cdots Z_{j_{h}} f\right\|_{L^{p, \lambda}\left(\mathbb{R}^{n+1}\right)}+\left\|\partial_{t}^{m} f\right\|_{L^{p, \lambda}\left(\mathbb{R}^{n+1}\right)}\right) .
\end{aligned}
$$

Moreover, we denote by $S_{0, Z}^{k, p, \lambda}\left(\mathbb{R}^{n+1}\right)$ the closed hull of $C_{0}^{\infty}\left(\mathbb{R}^{n+1}\right)$ with respect to the norm in $S_{Z}^{k, p, \lambda}\left(\mathbb{R}^{n+1}\right)$. Analogous definitions can be given for function spaces defined on a bounded domain $U \subset \mathbb{R}^{n+1}$.

In particular, we will denote by $S_{X}^{k, p, \lambda}$ and $S_{\tilde{X}}^{k, p, \lambda}$ the Sobolev-Morrey spaces generated by the original vector fields $X_{1}, \cdots X_{q}$ and the lifted $\tilde{X}_{1}, \cdots \tilde{X}_{q}$, respectively.

In this section, we consider the operator $H$ in (6.1). Under the assumptions (H1)-(H3) in Section 1, we have the following local estimate in the Sobolev-Morrey space $S_{X}^{2, p, \lambda}(U)$ for the operator $H$.

THeOREM 7.2. For every subdomain $U^{\prime} \subset \subset U \subset \mathbb{R}^{n+1}, p \in(1, \infty)$ and $\lambda \in\left(0, q^{\prime}\right)$, there exists a constant $c>0$ depending on $U, U^{\prime},\left\{X_{i}\right\}, p, \lambda, \mu$ and the VMO moduli $\left\|a_{i j}\right\|_{*}$ of $a_{i j}$ such that for every $f \in S_{X}^{2, p, \lambda}(U)$ with $H f \in L^{p, \lambda}(U)$, one has

$$
\|f\|_{S_{X}^{2, p, \lambda}\left(U^{\prime}\right)} \leq c\left\{\|H f\|_{L^{p, \lambda}(U)}+\|f\|_{L^{p, \lambda}(U)}\right\} .
$$

The proof of Theorem 7.2 needs some preliminary results. First, we recall the basic "representation formula"( see [2, Theorem 6.8]).

LEMma 7.3 (Parametrix for $\tilde{H}_{0}$ in (6.3)). For test functions $a, b$ and every $\left(t_{0}, \xi_{0}\right)$, there exist a frozen operator of type $2, P^{*}\left(t_{0}, \xi_{0}\right)$, and $q^{2}$ frozen operators of type $1, S_{i j}\left(t_{0}, \xi_{0}\right)(i, j=1 \cdots q)$, such that for every compactly supported function $f$,

$$
P^{*}\left(t_{0}, \xi_{0}\right) \tilde{H}_{0} f(t, \xi)=a(\xi) f(t, \xi)+\sum_{i, j=1}^{q} \tilde{a}_{i, j}\left(t_{0}, \xi_{0}\right) S_{i j}\left(t_{0}, \xi_{0}\right) f(t, \xi) .
$$


In particular,

$$
P^{*}\left(t_{0}, \xi_{0}\right) f(t, \xi)=\int_{-\infty}^{t} \int_{\mathbb{R}^{N}} a(\xi) b(\eta) \Gamma\left(t_{0}, \xi_{0} ; t-s, \Theta(\xi, \eta) f(s, \eta) d \eta d s .\right.
$$

Next, we prove $S_{0, \tilde{X}}^{2, p, \lambda}$ estimates for the operator $\tilde{H}$ in (6.2) when the function $f$ has a small support in $\mathbb{R}^{N+1}$.

THEOREM 7.4. There exist $r, c>0$ such that for any $f \in S_{0, \tilde{X}}^{2, p, \lambda}(\tilde{U})$ with $\operatorname{sprt} f \subset \tilde{B}_{r}\left(t_{0}, \xi_{0}\right) \subset \tilde{U} \subset \mathbb{R}^{N+1}$ and $\tilde{H} f \in L^{p, \lambda}(\tilde{U})$,

$$
\|f\|_{S_{0, \tilde{X}}^{2, p, \lambda}\left(\tilde{B}_{r}\right)} \leq c\left\{\|\tilde{H} f\|_{L^{p, \lambda}\left(\tilde{B}_{r}\right)}+\|f\|_{L^{p, \lambda\left(\tilde{B}_{r}\right)}}\right\} .
$$

where $r, c$ depend on $\left\{\tilde{X}_{i}\right\}, p, \lambda, \mu$ and $\left\|a_{i j}\right\|_{*}$.

Proof. Applying $\tilde{X}_{r} \tilde{X}_{s}$ to both sides of (7.1), and noting Lemma 6.4 and Lemma 6.5, we get

$\tilde{X}_{r} \tilde{X}_{s}(a f)(t, \xi)$

$=T\left(t_{0}, \xi_{0}\right) \tilde{H}_{0} f(t, \xi)-\sum_{i, j=1}^{q} \tilde{a}_{i j}\left(t_{0}, \xi_{0}\right)\left(\sum_{k=1}^{q} T_{i j}^{k}\left(t_{0}, \xi_{0}\right) \tilde{X}_{k} f(t, \xi)+T_{i j}^{0}\left(t_{0}, \xi_{0}\right) f(t, \xi)\right)$,

where $T\left(t_{0}, \xi_{0}\right), T_{i, j}^{k}\left(t_{0}, \xi_{0}\right)(k=0,1, \ldots, q)$ are frozen operators of type 0 . Denote $\tilde{H}_{0}=\tilde{H}+\left(\tilde{H}_{0}-\tilde{H}\right)$ and let $\left(t_{0}, \xi_{0}\right)=(t, \xi)$. Then

$$
\tilde{X}_{r} \tilde{X}_{s}(a f)(t, \xi)=T \tilde{H} f(t, \xi)+\sum_{i, j=1}^{q}\left[T\left(\tilde{a}_{i j}(\cdot) \tilde{X}_{i} \tilde{X}_{j} f\right)(t, \xi)-\tilde{a}_{i j}(t, \xi) T\left(\tilde{X}_{i} \tilde{X}_{j}\right) f(t, \xi)\right]
$$

$$
-\sum_{i, j=1}^{q} \tilde{a}_{i j}(t, \xi)\left(\sum_{k=1}^{q} T_{i j}^{k} \tilde{X}_{k} f(t, \xi)+T_{i j}^{0} f(t, \xi)\right)
$$

where $T(t, \xi), T_{i, j}^{k}(t, \xi)$ are variable operators of type 0 .

Now, for every $p \in(1, \infty), \lambda \in\left(0, Q^{\prime}\right)$, fixed $\varepsilon>0$ and test function $f$ with support small enough (depending $\varepsilon$ ), taking $L^{p, \lambda}\left(\tilde{B}_{r}\right)$ norm on both sides of (7.2) and using Theorem 6.6, we have

$$
\left\|\tilde{X}_{k} \tilde{X}_{h} f\right\|_{p, \lambda ; \tilde{B}_{r}} \leq c\|\tilde{H} f\|_{p, \lambda ; \tilde{B}_{r}}+\varepsilon \cdot \sum_{i, j=1}^{q}\left\|\tilde{X}_{i} \tilde{X}_{j} f\right\|_{p, \lambda ; \tilde{B}_{r}}+c \sum_{l=1}^{q}\left\|\tilde{X}_{l} f\right\|_{p, \lambda ; \tilde{B}_{r}}+c\|f\|_{p, \lambda ; \tilde{B}_{r}},
$$

that is,

$$
\left\|\tilde{X}_{k} \tilde{X}_{h} f\right\|_{p, \lambda ; \tilde{B}_{r}} \leq c\left\{\|\tilde{H} f\|_{p, \lambda ; \tilde{B}_{r}}+\sum_{l=1}^{q}\left\|\tilde{X}_{l} f\right\|_{p, \lambda ; \tilde{B}_{r}}+\|f\|_{p, \lambda ; \tilde{B}_{r}}\right\} .
$$


Note that the constant $c$ depends on the VMO moduli of $\tilde{a}_{i j}$, which are determined by the $V M O$ moduli of $a_{i j}$ by means of Theorem 5.4.

We want to remove the term $\left\|\tilde{X}_{l} f\right\|_{p, \lambda ; \tilde{B}_{r}}$ in the right-hand side of (7.3). To do this, we come back to (7.1) with $a(\xi)=1$ and take only one derivative $\tilde{X}_{l}$ to obtain

$$
\begin{aligned}
& \tilde{X}_{l} f(\xi)=T \tilde{H} f(t, \xi) \\
& -\sum_{i, j=1}^{q}\left[T\left(\tilde{a}_{i j}(\cdot) \tilde{X}_{i} \tilde{X}_{j} f\right)(t, \xi)-\tilde{a}_{i j}(t, \xi) T\left(\tilde{X}_{i} \tilde{X}_{j} f\right)(t, \xi)\right]-\sum_{i, j=1}^{q} \tilde{a}_{i j}(t, \xi) T^{i j} f(t, \xi),
\end{aligned}
$$

where $T$ is a variable operator of type 1 , and $T^{i j}$ are variable operators of type 0 . Therefore,

$$
\left\|\tilde{X}_{l} f\right\|_{p, \lambda ; \tilde{B}_{r}} \leq c\|\tilde{H} f\|_{p, \lambda ; \tilde{B}_{r}}+\varepsilon \cdot \sum_{i, j=1}^{q}\left\|\tilde{X}_{i} \tilde{X}_{i} f\right\|_{p, \lambda ; \tilde{B}_{r}}+c\|f\|_{p, \lambda ; \tilde{B}_{r}} .
$$

Substituting this in (7.3),

$$
\left\|\tilde{X}_{k} \tilde{X}_{h} f\right\|_{p, \lambda ; \tilde{B}_{r}} \leq c\left\{\|\tilde{H} f\|_{p, \lambda ; \tilde{B}_{r}}+\|f\|_{p, \lambda ; \tilde{B}_{r}}\right\} .
$$

From (6.2) we also read

$$
\left\|\partial_{t} f\right\|_{p, \lambda ; \tilde{B}_{r}} \leq\|\tilde{H} f\|_{p, \lambda ; \tilde{B}_{r}}+c\left\|\tilde{X}_{k} \tilde{X}_{h} f\right\|_{p, \lambda ; \tilde{B}_{r}} .
$$

Combining two estimates above yields

$$
\|f\|_{S_{\tilde{X}}^{2, p, \lambda}\left(\tilde{B}_{r}\right)} \leq c\left\{\|\tilde{H} f\|_{L^{p, \lambda\left(\tilde{B}_{r}\right)}}+\|f\|_{L^{p, \lambda}\left(\tilde{B}_{r}\right)}\right\} .
$$

Furthermore, we prove the estimate of $\tilde{H}$ in $S_{\tilde{X}}^{2, p, \lambda}$ when the function $f$ has no the restriction of compact support.

THEOREM 7.5. Let $\tilde{U}^{\prime} \subset \subset \tilde{U} \subset \mathbb{R}^{N+1}$ be a bounded domain. If $f \in S_{\tilde{X}}^{2, p, \lambda}(\tilde{U})$ with $\tilde{H} f \in L^{p, \lambda}(\tilde{U})$, then

$$
\|f\|_{S_{\tilde{X}}^{2, \lambda, \lambda}\left(\tilde{U}^{\prime}\right)} \leq c\left\{\|\tilde{H} f\|_{L^{p, \lambda}(\tilde{U})}+\|f\|_{L^{p, \lambda}(\tilde{U})}\right\},
$$

where $c$ depends on $\left\{\tilde{X}_{i}\right\}, p, \lambda, \mu, s, t$ and $\left\|a_{i j}\right\|_{*}$.

In order to prove Theorem 7.5, we establish some interpolation inequalities.

Proposition 7.6 (Interpolation for test function). Let $\mathbb{H}=\partial_{t}-\sum \tilde{X}_{i}^{2}$. For every $R>0, p \in(1, \infty)$ and $\lambda \in\left(0, Q^{\prime}\right)$, for every $\varepsilon>0$ there exist 
positive constants $c(\varepsilon)$ such that for any $f \in C_{0}^{\infty}\left(\tilde{B}_{R}\right)$,

$$
\left\|\tilde{X}_{i} f\right\|_{p, \lambda ; \tilde{B}_{R}} \leq \varepsilon\|H f\|_{p, \lambda ; \tilde{B}_{R}}+c(\varepsilon)\|f\|_{p, \lambda ; \tilde{B}_{R}} \text {, for every } i=1, \cdots, q .
$$

Proof. By Lemma 7.3, we write

$$
P_{2} \mathbb{H} f(t, \xi)=a(\xi) f(t, \xi)+S_{1} f(t, \xi),
$$

where $P_{2}$ is a constant operator of type $2, S_{1}$ is a constant operator of type 1 and $a(\xi)$ is a test function. We take $a(\xi) \equiv 1$ on $\tilde{B}_{R}$, then for $f \in C_{0}^{\infty}\left(\tilde{B}_{R}\right)$,

$$
f(t, \xi)=P_{2} H f(t, \xi)-S_{1} f(t, \xi) .
$$

Applying $\tilde{X}_{i}$ to both sides of (7.4) shows

$$
\tilde{X}_{i} f(\xi)=P_{1} H f(\xi)+S_{0} f(\xi),
$$

where $P_{1}$ is a constant operator of type 1 , and $S_{0}$ is a constant operator of type 0 . Hence we need to show that

$$
\left\|P_{1} \mathbb{H} f\right\|_{p, \lambda ; \tilde{B}_{R}} \leq \varepsilon\|\mathbb{H} f\|_{p, \lambda ; \tilde{B}_{R}}+c(\varepsilon)\|f\|_{p, \lambda ; \tilde{B}_{R}} .
$$

Set

$$
P_{1} \mathbb{H} f=\int_{\tilde{B}_{R}} k(t, \xi, s, \eta) H(f(s, \eta) d \eta d s,
$$

with $k$ being the kernel of $P_{1}$, and let $\varphi_{\varepsilon}$ be a cutoff function such that $\tilde{B}_{\varepsilon / 2}(t, \xi) \prec \varphi_{\varepsilon} \prec \tilde{B}_{\varepsilon}(t, \xi)$. We split $P_{1} \mathrm{H}$ into:

$$
\begin{aligned}
P_{1} \mathbb{H} f(t, \xi)= & \int_{\tilde{d}^{\prime}((s, \eta),(t, \xi))>\varepsilon / 2} k(t, \xi, s, \eta)\left[1-\varphi_{\varepsilon}(s, \eta)\right] \mathrm{H} f(s, \eta) d \eta d s \\
& +\int_{\tilde{d}^{\prime}((s, \eta),(t, \xi)) \leq \varepsilon} k(t, \xi, s, \eta) \varphi_{\varepsilon}(s, \eta) H[H(s, \eta) d \eta d s \\
= & : I_{\varepsilon}+I I_{\varepsilon} .
\end{aligned}
$$

Then, writting

$$
\begin{aligned}
\left|I_{\varepsilon}\right| & =\int_{\tilde{d}^{\prime}((s, \eta),(t, \xi))>\varepsilon / 2} \mathbb{H}^{T}\left[k(t, \xi, \cdot, \cdot)\left(1-\varphi_{\varepsilon}(\cdot, \cdot)\right)\right](s, \eta) f(s, \eta) d \eta d s \\
& \leq \int_{\tilde{d}^{\prime}((s, \eta),(t, \xi))>\varepsilon / 2}\left[\left|\mathbb{H}^{T} k(t, \xi, \cdot, \cdot)\right|+\left|D k D \varphi_{\varepsilon}\right|+\left|k \mathbb{H}^{T} \varphi_{\varepsilon}\right|\right] f(s, \eta) d \eta d s,
\end{aligned}
$$


where $\mathbb{H}^{T}$ denotes the transpose of $H$, we see that both $I_{\varepsilon}$ and $I I_{\varepsilon}$ satisfy $L^{p}$ estimates (see [1]). By Lemma 6.5, Lemma 3.1 and Theorem 3.2, we have

$$
\left\|I_{\varepsilon}\right\|_{p, \lambda ; \tilde{B}_{R}} \leq c(\varepsilon)\|f\|_{p, \lambda ; \tilde{B}_{R}}, \quad\left\|I I_{\varepsilon}\right\|_{p, \lambda ; \tilde{B}_{R}} \leq c \varepsilon\|\mathrm{H} f\|_{p, \lambda ; \tilde{B}_{R}} .
$$

Proposition 7.7 (Interpolation inequality for not necessary test function). There exist positive constants $c, R$ such that for any $f \in S_{\tilde{X}}^{2, p, \lambda}\left(\tilde{B}_{R}\right)$, $0<\rho<R, 0<\delta<1 / 3$,

$$
\|D f\|_{p, \lambda ; \tilde{B}_{\rho}} \leq \delta\left[\left\|D^{2} f\right\|_{p, \lambda ; \tilde{B}_{R}}+\left\|\partial_{t} f\right\|_{p, \lambda ; \tilde{B}_{R}}\right]+c(\delta)\|f\|_{p, \lambda ; \tilde{B}_{R}},
$$

here $D$ is the differential operator such that for $s \geq 1, D^{s} f=\tilde{X}_{i_{1}} \tilde{X}_{i_{2}} \cdots \tilde{X}_{i_{s}} f$, for any $1 \leq i_{j} \leq q, 1 \leq j \leq s$.

Proof. Let $\varphi$ be a cutoff function such that $\tilde{B}_{t} \prec \varphi \prec \tilde{B}_{s}$. Applying Proposition 7.6 to $f \varphi$ leads to

$$
\|D f\|_{p, \lambda ; \tilde{B}_{t}} \leq\|D(f \varphi)\|_{p, \lambda ; \tilde{B}_{s}} \leq \varepsilon\|\mathbb{H}(f \varphi)\|_{p, \lambda ; \tilde{B}_{s}}+c(\varepsilon)\|f\|_{p, \lambda ; \tilde{B}_{s}} .
$$

By Lemma 6.5,

$$
\|D f\|_{p, \lambda ; \tilde{B}_{t}} \leq \varepsilon\|\mathbb{H} f\|_{p, \lambda ; \tilde{B}_{s}}+\frac{c \varepsilon}{(s-t)}\|D f\|_{p, \lambda ; \tilde{B}_{s}}+\frac{c(\varepsilon)}{(s-t)^{2}}\|f\|_{p, \lambda ; \tilde{B}_{s}} .
$$

Choosing $\varepsilon=\delta(s-t) / c$ and using Lemma 7.3 in [2],

$$
\begin{aligned}
\|D f\|_{p, \lambda ; \tilde{B}_{t}} & \leq c_{1} \delta\|\mathrm{H} f\|_{p, \lambda ; \tilde{B}_{s}}+c_{1}(\delta)\|f\|_{p, \lambda ; \tilde{B}_{s}} \\
& \leq c_{1} \delta\left[\left\|D^{2} f\right\|_{p, \lambda ; \tilde{B}_{R}}+\left\|\partial_{t} f\right\|_{p, \lambda ; \tilde{B}_{R}}\right]+c_{1}(\delta)\|f\|_{p, \lambda ; \tilde{B}_{s}},
\end{aligned}
$$

where $c_{1}$ depends on $\varepsilon, s, t$.

Proof of Theorem 7.5. - If $f \in S_{\tilde{X}}^{2, p, \lambda}\left(\tilde{B}_{r}\right)$, with $r$ small enough to use Theorem 7.4, $t<r, s=(t+r) / 2$, and $\varphi$ is a cutoff function such that $\tilde{B}_{t} \prec \varphi \prec \tilde{B}_{s}$, then we apply Theorem 7.4 to $f \varphi$,

$$
\left\|\tilde{X}_{i} \tilde{X}_{j} f\right\|_{p, \lambda ; \tilde{B}_{t}} \leq c\left(\|\tilde{H} f \varphi\|_{p, \lambda ; \tilde{B}_{s}}+\|D f D \varphi\|_{p, \lambda ; \tilde{B}_{s}}+\|f \tilde{H} \varphi\|_{p, \lambda ; \tilde{B}_{s}}\right) .
$$

By Lemma 6.5 and Proposition 7.7,

$$
\left\|\tilde{X}_{i} \tilde{X}_{j} f\right\|_{p, \lambda ; \tilde{B}_{t}} \leq c\left(\|\tilde{H} f\|_{p, \lambda ; \tilde{B}_{s}}+\left\|\tilde{X}_{i} \tilde{X}_{j} f\right\|_{p, \lambda ; \tilde{B}_{R}}+\left\|\partial_{t} f\right\|_{p, \lambda ; \tilde{B}_{R}}+\|f\|_{p, \lambda ; \tilde{B}_{R}}\right) .
$$

Therefore,

$$
\|f\|_{S_{\tilde{X}}^{2, p, \lambda}\left(\tilde{B}_{t}\right)} \leq c\left\{\|\tilde{H} f\|_{L^{p, \lambda}\left(\tilde{B}_{s}\right)}+\|f\|_{L^{p, \lambda}\left(\tilde{B}_{s}\right)}\right\} .
$$


Making the similar argument as in Theorem 5.1 of [2], we reach the proof of Theorem 7.5.

Proof of Theorem 7.2. - Applying the same lifting technique as in [1, Theorem 0.1] and Theorem 7.5 here, we can complete the proof.

\section{Morrey estimates in $S_{X}^{k+2, p, \lambda}(U)$.}

In this section we plan to extend Theorem 7.2 to higher order derivatives and prove Theorem 1.1 for the operator without lower order terms. We will use the way of Section 7.

THEOREM 8.1. Let $k$ be a nonnegative integer and the coefficients $a_{i j} \in S_{X}^{k, \infty, 0}(U)$ satisfy the assumptions (H2)-(H3). For every subdomain $U^{\prime} \subset \subset U \subset \mathbb{R}^{n+1}, p \in(1, \infty)$ and $\lambda \in\left(0, q^{\prime}\right)$, there exists a constant $c>0$ depending on $U, U^{\prime},\left\{X_{i}\right\}, p, \lambda, \mu$ and $\left\|a_{i j}\right\|_{*}$, such that for every $f \in S_{X}^{k+2, p, \lambda}(U)$ with $H f \in S_{X}^{k, p, \lambda}(U)$, one has

$$
\|f\|_{S_{X}^{k+2, p, \lambda}\left(U^{\prime}\right)} \leq c\left\{\|H f\|_{S_{X}^{k, p, \lambda}(U)}+\|f\|_{L^{p, \lambda}(U)}\right\} .
$$

Let us recall the definition of Sobolev-Morrey norm:

$$
\|f\|_{S_{X}^{k, p, \lambda}(U)}=\sum_{|I|+2 h \leq k}\left\|\partial_{t}^{h} D^{I}\right\|_{L^{p, \lambda}(U)},
$$

where $D^{I} f=X_{i_{1}} X_{i_{2}} \cdots X_{i_{s}} f$ for any multiindex $I=\left(i_{1}, i_{2}, \ldots i_{s}\right)$ with $1 \leq i_{j} \leq q$, and $|I|=s$.

To prove Theorem 8.1, we first get the analog of Theorem 7.4 for $\|f\|_{S_{\tilde{X}}^{k+2, p, \lambda}\left(\tilde{B}_{r}\right)^{*}}$

THEOREM 8.2. Under assumptions of Theorem 8.1, there exists $r>0$ such that for any $f \in S_{0, \tilde{X}}^{k+2, p, \lambda}\left(\tilde{B}_{r}\right)$ with $\tilde{H} f \in S_{0, \tilde{X}}^{k, p, \lambda}\left(\tilde{B}_{r}\right)$, and $\tilde{B}_{r}=\tilde{B}_{r}\left(t_{0}, \xi_{0}\right) \subset \tilde{U}$,

$$
\|f\|_{S_{\tilde{X}}^{k+2, p, \lambda}\left(\tilde{B}_{r}\right)} \leq c\left\{\|\tilde{H} f\|_{S_{\tilde{X}}^{k, p, \lambda\left(\tilde{B}_{r}\right)}}+\|f\|_{L^{p, \lambda\left(\tilde{B}_{r}\right)}}\right\},
$$

where $r, c>0$ depend on $\left\{\tilde{X}_{i}\right\}, p, \lambda, \mu$ and $\left\|a_{i j}\right\|_{*}$.

The proof of this theorem will be achieved through the following two lemmas. 
LEMMA 8.3. Let $\tilde{a}_{i j} \in S_{\tilde{X}}^{k, \infty, 0}\left(\tilde{B}_{r}\right)$. For every integer $k \geq 0$, there exists $r>0$ such that for every $f \in S_{0, \tilde{X}}^{k+2, p, \lambda}\left(\tilde{B}_{r}\right)$ with $\tilde{H} f \in S_{0, \tilde{X}}^{k, p, \lambda}\left(\tilde{B}_{r}\right)$,

$$
\left\|D^{k+2} f\right\|_{p, \lambda ; \tilde{B}_{r}} \leq c\left(\left\|D^{k} \tilde{a}_{i j}\right\|_{L^{\infty}} \sum_{h \leq k+1}\left\|D^{h} f\right\|_{p, \lambda ; \tilde{B}_{r}}+\|\tilde{H} f\|_{S_{\tilde{X}}^{k, p, \lambda}\left(\tilde{B}_{r}\right)}\right)
$$

and

$$
\left\|D^{k+2} f\right\|_{p, \lambda ; \tilde{B}_{r}} \leq c\left(\|f\|_{p, \lambda ; \tilde{B}_{r}}+\|\tilde{H} f\|_{S_{\tilde{X}}^{k, p, \lambda}\left(\tilde{B}_{r}\right)}\right)
$$

where c depends on $\left\|D^{k} \tilde{a}_{i j}\right\|_{L^{\infty}}$, and the derivative here is in the sense of distributions.

Proof. From Lemma 9.4 in [2], we know for any multiindex $J,|J|=k$, and $m, l=1, \cdots, q$,

$$
\begin{aligned}
& \tilde{X}_{m} \tilde{X}_{l} \tilde{X}^{J}(a f) \\
& =\sum_{h=0}^{k} \sum_{|I|=h}\left\{\left[T_{I}, \tilde{a}_{i j}\right] \tilde{X}^{I} \tilde{X}_{i} \tilde{X}_{j} f-T_{I}\left[\sum_{\left|J^{\prime}\right|+\left|J^{\prime \prime}\right|=|I|} \tilde{X}^{J^{\prime}} \tilde{a}_{i j} \tilde{X}^{J^{\prime \prime}} \tilde{X}_{i} \tilde{X}_{j} f+\tilde{X}^{I} \tilde{H} f\right]\right. \\
& \left.+\sum_{h=1}^{q} T_{I, h} \tilde{X}_{h} \tilde{X}^{I} f+T_{I, 0} \tilde{X}^{I} f\right\},
\end{aligned}
$$

for suitable operators $T_{I}, T_{I, h}, T_{I, 0}$ of type 0 . By Theorem 6.6,

$$
\begin{aligned}
& \left\|\tilde{X}_{m} \tilde{X}_{l} \tilde{X}^{J}(a f)\right\|_{p, \lambda ; \tilde{B}_{r}} \\
& \leq c \sum_{h=0}^{k} \sum_{|I|=h}\left\{\left\|\tilde{X}^{I} \tilde{X}_{i} \tilde{X}_{j} f\right\|_{p, \lambda ; \tilde{B}_{r}}+\sum_{\left|J^{\prime}\right|+\left|J^{\prime \prime}\right|=|I|}\left\|\tilde{X}^{J^{\prime}} \tilde{a}_{i j}\right\|_{L^{\infty}}\left\|\tilde{X}^{J^{\prime \prime}} \tilde{X}_{i} \tilde{X}_{j} f\right\|_{p, \lambda ; \tilde{B}_{r}}\right. \\
& \left.\quad+\left\|\tilde{X}^{I} \tilde{H} f\right\|_{p, \lambda ; \tilde{B}_{r}}+\sum_{h=1}^{q}\left\|\tilde{X}_{h} \tilde{X}^{I} f\right\|_{p, \lambda ; \tilde{B}_{r}}+\left\|\tilde{X}^{I} f\right\|_{p, \lambda ; \tilde{B}_{r}}\right\} .
\end{aligned}
$$

Proposition 7.6 and the similar argument as in Theorem 7.4 give $\left\|D^{k+2} f\right\|_{p, \lambda ; \tilde{B}_{r}} \leq c\left(\varepsilon\left\|D^{k+2} f\right\|_{p, \lambda ; \tilde{B}_{r}}+\left\|D^{k} \tilde{a}_{i j}\right\|_{L^{\infty}} \sum_{h \leq k+1}\left\|D^{h} f\right\|_{p, \lambda ; \tilde{B}_{r}}+\sum_{h \leq k}\left\|D^{h} \tilde{H} f\right\|_{p, \lambda ; \tilde{B}_{r}}\right)$.

This completes the proof of (8.2). Then we conclude (8.3) by iteration.

LEMma 8.4. For any triple of integers $k, h, m$ such that $k \geq 1, h \geq 1$, $m \geq 0,2 h+m \leq k+2$, we have

$$
\left\|\partial_{t}^{h} D^{m} f\right\|_{p, \lambda ; \tilde{B}_{r}} \leq c_{a, k}\left(\|\tilde{H} f\|_{S_{\tilde{X}}^{k, p, \lambda}\left(\tilde{B}_{r}\right)}+\|f\|_{p, \lambda ; \tilde{B}_{r}}\right) .
$$


Proof. We will prove (8.4) by induction on $h$. For $h=1$ we have to show that

$$
\sum_{m=0}^{k}\left\|\partial_{t} D^{m} f\right\|_{p, \lambda ; \tilde{B}_{r}} \leq c_{a, k}\left(\|\tilde{H} f\|_{S_{\tilde{X}}^{k, p, \lambda}\left(\tilde{B}_{r}\right)}+\|f\|_{p, \lambda ; \tilde{B}_{r}}\right) .
$$

In fact, by (6.2) and (8.3),

$$
\begin{aligned}
\left\|\partial_{t} D^{m} f\right\|_{p, \lambda ; \tilde{B}_{r}} & \leq c\left(\|\tilde{H} f\|_{S_{\tilde{X}}^{k, p, \lambda}\left(\tilde{B}_{r}\right)}+\sum_{i, j=1}^{q} \sum_{l=0}^{m}\left\|D^{m-l} \tilde{a}_{i j}\right\|_{L^{\infty}}\left\|D^{l+2} f\right\|_{p, \lambda ; \tilde{B}_{r}}\right) \\
& \leq c_{a, k}\left(\|\tilde{H} f\|_{S_{\tilde{X}}^{k, p, \lambda}\left(\tilde{B}_{r}\right)}+\|f\|_{p, \lambda ; \tilde{B}_{r}}\right) .
\end{aligned}
$$

Assume (8.4) holds up to $h-1$. From inductive hypothesis we get

$$
D^{m} \partial_{t}^{h} f=D^{m} \partial_{t}^{h-1} \tilde{H} f+D^{m} \partial_{t}^{h-1}\left(\sum_{i, j=1}^{q} \tilde{a}_{i j} \tilde{X}_{i} \tilde{X}_{j} f\right),
$$

then

$$
\begin{aligned}
\left\|D^{m} \partial_{t}^{h} f\right\|_{p, \lambda ; \tilde{B}_{r}} & \leq\|\tilde{H} f\|_{S_{\tilde{X}}^{m+2 h-2, p, \lambda,\left(\tilde{B}_{r}\right)}}+c_{a, m+2 h-2} \sum_{l=0}^{m}\left\|D^{l+2} \partial_{t}^{h-1} f\right\|_{p, \lambda ; \tilde{B}_{r}} \\
& \leq c_{a, k}\left(\|\tilde{H} f\|_{S_{\tilde{X}}^{k, p, \lambda}\left(\tilde{B}_{r}\right)}+\|f\|_{p, \lambda ; \tilde{B}_{r}}\right) .
\end{aligned}
$$

Proof of Theorem 8.2. - Uniting Lemma 8.3 and Lemma 8.4 implies the desired result.

We can prove Theorem 8.1 by iterating results in Lemma 8.3 and Lemma 8.4. This process can be performed as in the proof of Theorem 9.6 in [2]. Since the proof is tedious, we only state the sketch.

Proof OF Theorem 8.1 (Sketch). - Let $f \in S_{\tilde{X}}^{k+2, p, \lambda}\left(\tilde{B}_{s}\right)$ and $\varphi$ be a cutoff function such that $\tilde{B}_{t} \prec \varphi \prec \tilde{B}_{s}$. Applying (8.2) to $f \varphi$, by Lemma 6.5, we get

$$
\left\|D^{k+2} f\right\|_{p, \lambda ; \tilde{B}_{t}} \leq c_{a, k}\left(\sum_{h \leq k+1}\left\|D^{h} f\right\|_{p, \lambda ; \tilde{B}_{s}}+\|\tilde{H} f\|_{S_{\tilde{X}}^{k, p, \lambda}\left(\tilde{B}_{s}\right)}\right) .
$$

To consider the terms involving time derivatives, we apply (8.4) to $f \varphi$. Using Lemma 6.5 yields

$$
\left\|\partial_{t}^{h} D^{m} f\right\|_{p, \lambda ; \tilde{B}_{t}} \leq c_{a, k}\left(\|\tilde{H} f\|_{S_{\tilde{X}}^{k, p, \lambda}\left(\tilde{B}_{s}\right)}+\|f\|_{S_{\tilde{X}}^{k+1, p, \lambda}\left(\tilde{B}_{s}\right)}\right),
$$

for any triple of integers $k, h, m, k \geq 1, h \geq 1, m \geq 0,2 h+m \leq k+2$. 
Combining (8.6) with (8.7), and using Proposition 7.7 and iteration, we write

$$
\|f\|_{S_{\tilde{X}}^{k+2, p, \lambda}\left(\tilde{B}_{t}\right)} \leq c\left\{\|\tilde{H} f\|_{S_{\tilde{X}}^{k, p, \lambda}\left(\tilde{B}_{s}\right)}+\|f\|_{L^{p, \lambda}\left(\tilde{B}_{s}\right)}\right\} .
$$

Reasoning like the proof of Theorem 5.1 in [2], we finally complete the proof of Theorem 8.1 .

\section{Local estimate for the operator (1.5).}

In this section, for fulfilling the proof of Theorem 1.1, we start with $S_{X}^{2, p, \lambda}$ estimate.

THEOREM 9.1. Under assumptions of Theorem 1.1, for every subdomain $U^{\prime} \subset \subset U$, there exists a constant $c>0$ such that for every $f \in S_{X}^{2, p, \lambda}(U)$ with $H_{1} f \in L^{p, \lambda}(U)$, one has

$$
\|f\|_{S_{X}^{2, p, \lambda}\left(U^{\prime}\right)} \leq c\left\{\left\|H_{1} f\right\|_{L^{p, \lambda}(U)}+\|f\|_{L^{p, \lambda}(U)}\right\},
$$

where $c$ depends on $U, U^{\prime}\left\{X_{i}\right\}, p, \lambda, \mu$ and suitable modulus of the coefficients $a_{i j}, b_{j}, c$.

The proof of Theorem 9.1 is based on the following proposition.

Proposition 9.2. If coefficients $b_{j}(j=1, \ldots, q)$ and c satisfy conditions of Theorem 1.1, then their lifted analogs $\tilde{b}_{j}$ and $\tilde{c}$ satisfy:

$\tilde{b}_{j} \in\left\{\begin{array}{l}L^{Q} \cap S_{\tilde{X}}^{k, Q, 0}(\tilde{U}), p+\lambda \leq Q^{\prime} ; \\ L^{p, \lambda} \cap S_{\tilde{X}}^{k, p, \lambda}(\tilde{U}), p+\lambda>Q^{\prime},\end{array} \quad \tilde{c} \in\left\{\begin{array}{l}L^{Q / 2} \cap S_{\tilde{X}}^{k, Q / 2,0}(\tilde{U}), 2 p+\lambda \leq Q^{\prime} ; \\ L^{p, \lambda} \cap S_{\tilde{X}}^{k, p, \lambda}(\tilde{U}), 2 p+\lambda>Q^{\prime},\end{array}\right.\right.$

where $k$ is a positive integer. Moreover, for every $f \in S_{0, \tilde{X}}^{k+2, p, \lambda}(\tilde{U})$ with sprt $f \subset \tilde{B}_{r}$, one has

$$
\begin{gathered}
\left\|\tilde{b}_{j} \tilde{X}_{j} f\right\|_{S_{\tilde{X}}^{k, p, \lambda}\left(\tilde{B}_{r}\right)} \leq\left\{\begin{array}{l}
C\left\|\tilde{b}_{j}\right\|_{S_{\tilde{X}}^{k, Q, 0}\left(\tilde{B}_{r}\right)}\|f\|_{S_{\tilde{X}}^{k+2, p, \lambda}\left(\tilde{B}_{r}\right)}, p+\lambda \leq Q^{\prime} ; \\
C\left\|\tilde{b}_{j}\right\|_{S_{\tilde{X}}^{k, p, \lambda}\left(\tilde{B}_{r}\right)}\|f\|_{S_{\tilde{X}}^{k+2, p, \lambda}\left(\tilde{B}_{r}\right)}, p+\lambda>Q^{\prime} .
\end{array}\right. \\
\|\tilde{c} f\|_{S_{\tilde{X}}^{k, p, \lambda}\left(\tilde{B}_{r}\right)} \leq\left\{\begin{array}{l}
C\|\tilde{c}\|_{S_{\tilde{X}}^{k, Q / 2,0}\left(\tilde{B}_{r}\right)}\|f\|_{S_{\tilde{X}}^{k, p, \lambda}\left(\tilde{B}_{r}\right)}, 2 p+\lambda \leq Q^{\prime} ; \\
C\|\tilde{c}\|_{S_{\tilde{X}}^{k, p, \lambda}\left(\tilde{B}_{r}\right)}\|f\|_{S_{\tilde{X}}^{k, p, \lambda}\left(\tilde{B}_{r}\right)}, 2 p+\lambda>Q^{\prime} .
\end{array}\right.
\end{gathered}
$$


Proof. It is easily seen from Lemma 5.3 that $\tilde{b}_{j}$ and $\tilde{c}$ belong to the corresponding Sobolev-Morrey spaces. If $p+\lambda \leq Q^{\prime}$, then Hölder's inequality and Sobolev type inequality on homogeneous groups (see [15]) imply

$$
\begin{aligned}
& \sum_{m \leq k}\left(\rho^{-\lambda} \int_{\tilde{B}_{r} \cap \tilde{B}_{\rho}}\left|D^{m}\left(\tilde{b}_{j} \tilde{X}_{j} f\right)\right|^{p}\right)^{1 / p} \leq C \sum_{m \leq k} \sum_{l \leq m} \rho^{-\lambda / p}\left(\int_{\tilde{B}_{r} \cap \tilde{B}_{\rho}}\left|D^{l} \tilde{b}_{j} D^{m-l}\left(\tilde{X}_{j} f\right)\right|^{p}\right)^{1 / p} \\
& \quad \leq C \sum_{m \leq k} \sum_{l \leq m}\left\|D^{l} \tilde{b}_{j}\right\|_{L^{Q}\left(\tilde{B}_{r}\right)} \rho^{-\lambda / p}\left\|D^{m-l+1} f\right\|_{L^{Q p}{ }^{Q-p}\left(\tilde{B}_{r}\right)} \\
& \quad \leq C \sum_{m \leq k} \sum_{l \leq m}\left\|D^{l} \tilde{b}_{j}\right\|_{L^{Q}\left(\tilde{B}_{r}\right)} \rho^{-\lambda / p}\left\|D^{m-l+1} f\right\|_{S_{\tilde{X}}^{1, p, 0}\left(\tilde{B}_{r}\right)} \\
& \quad \leq C\left\|\tilde{b}_{j}\right\|_{S_{\tilde{X}}^{k, Q, 0}\left(\tilde{B}_{r}\right)}\|f\|_{S_{\tilde{X}}^{k+2, p, \lambda}\left(\tilde{B}_{r}\right)} .
\end{aligned}
$$

If $p+\lambda>Q^{\prime}$, then we have from $S_{\tilde{X}}^{1, p, 0}\left(\tilde{B}_{r}\right) \hookrightarrow C^{0}\left(\tilde{B}_{r}\right)$,

$$
\begin{aligned}
& \sum_{m \leq k}\left(\rho^{-\lambda} \int_{\tilde{B}_{r} \cap \tilde{B}_{\rho}}\left|D^{m}\left(\tilde{b}_{j} f\right)\right|^{p}\right)^{1 / p} \\
& \leq C \sum_{m \leq k} \sum_{l \leq m}\left(\rho^{-\lambda} \int_{\tilde{B}_{r} \cap \tilde{B}_{\rho}}\left|D^{l} \tilde{b}_{j}\right|^{p}\right)^{1 / p} \sup _{\tilde{B}_{r}}\left|D^{m-l}\left(\tilde{X}_{j} f\right)\right| \\
& \leq C \sum_{m \leq k} \sum_{l \leq m}\left\|D^{l} \tilde{b}_{j}\right\|_{\left.L^{p, \lambda}, \tilde{B}_{r}\right)}|| D^{m-l+1} f \|_{S_{\tilde{X}}^{1, p, 0}\left(\tilde{B}_{r}\right)} \\
& \leq C\left\|\tilde{b}_{j}\right\|_{S_{\tilde{X}}^{k, p, \lambda}\left(\tilde{B}_{r}\right)}\|f\|_{S_{\tilde{X}}^{k+2, p, \lambda}\left(\tilde{B}_{r}\right)}
\end{aligned}
$$

Hence, (9.2) is proved. The examination of (9.3) is similar.

Proof OF THEOREM 9.1. - The proof will follow the same process in the proof of Theorem 7.2. Let

$$
\tilde{H}_{1}=\partial_{t}-\sum_{i, j=1}^{q} \tilde{a}_{i j}(t, \xi) \tilde{X}_{i} \tilde{X}_{j}-\sum_{j=1}^{q} \tilde{b}_{j}(t, \xi) \tilde{X}_{j}-\tilde{c}(t, \xi)=\tilde{H}-\sum_{j=1}^{q} \tilde{b}_{j}(t, \xi) \tilde{X}_{j}-\tilde{c}(t, \xi) .
$$

By (7.3) and Proposition 9.2, for every $f \in S_{0, \tilde{X}}^{2, p, \lambda}\left(\tilde{B}_{r}\right)$, with $r$ small enough, $\left\|\tilde{X}_{k} \tilde{X}_{h} f\right\|_{p, \lambda ; \tilde{B}_{r}} \leq C\left\{\left\|\tilde{H}_{1} f\right\|_{p, \lambda ; \tilde{B}_{r}}+\varepsilon\left\|D^{2} f\right\|_{p, \lambda ; \tilde{B}_{r}}+\sum_{l=1}^{q}\left\|\tilde{X}_{l} f\right\|_{p, \lambda ; \tilde{B}_{r}}+\|f\|_{p, \lambda ; \tilde{B}_{r}}\right\}$, 
where $\varepsilon>0$ depends on suitable moduli of $\tilde{b}_{j}, \tilde{c}$. To remove the term containing $\tilde{X}_{l} f$, we use the interpolation inequality in Proposition 7.7 and get

$$
\|f\|_{S_{0, \tilde{X}}^{2, p, \lambda}\left(\tilde{B}_{r}\right)} \leq C\left\{\left\|\tilde{H}_{1} f\right\|_{p, \lambda ; \tilde{B}_{r}}+\|f\|_{p, \lambda ; \tilde{B}_{r}}\right\} \text {. }
$$

As in the proof of Theorem 7.5, if $f \in S_{\tilde{X}}^{2, p, \lambda}(\tilde{U}), \tilde{B}_{r} \subset \tilde{U}, t<R$, $s=(t+R) / 2, \varphi$ is a cutoff function with $\tilde{B}_{t} \prec \varphi \prec \tilde{B}_{s}$, then by (9.4),

$$
\|f\|_{S_{\tilde{X}}^{2, p, \lambda}\left(\tilde{B}_{t}\right)} \leq C\left\{\left\|\tilde{H}_{1}(f \varphi)\right\|_{p, \lambda ; \tilde{B}_{s}}+\|f \varphi\|_{p, \lambda ; \tilde{B}_{s}}\right\} .
$$

Repeating the steps in the proof of Theorem 7.5 without changes, we have

$$
\|f\|_{S_{\tilde{X}}^{2, p, \lambda}\left(\tilde{U}^{\prime}\right)} \leq C\left\{\left\|\tilde{H}_{1} f\right\|_{p, \lambda ; \tilde{U}}+\|f\|_{p, \lambda ; \tilde{U}}\right\} .
$$

Finally, the same lifting method as in [1, Theorem 0.1] implies the estimate in (9.1).

Next, we extend Theorem 9.1 to the estimates for higher order derivatives and prove Theorem 1.1.

Proof of Theorem 1.1. From Theorem 8.2 and Proposition 9.2, we write for every $f \in S_{0, \tilde{X}}^{k+2, p, \lambda}\left(\tilde{B}_{r}\right)$ with $r$ small enough,

$$
\|f\|_{S_{\tilde{X}}^{k+2, p, \lambda}\left(\tilde{B}_{r}\right)} \leq C\left\{\left\|\tilde{H}_{1} f\right\|_{S_{\tilde{X}}^{k, p, \lambda}\left(\tilde{B}_{r}\right)}+\varepsilon\|f\|_{S_{\tilde{X}}^{k+2, p, \lambda}\left(\tilde{B}_{r}\right)}+\|f\|_{p, \lambda ; \tilde{B}_{r}}\right\} .
$$

where $\varepsilon>0$ depends on suitable moduli of $\tilde{b}_{j}, \tilde{c}$. The discussion as in the proof of Theorem 9.1 shows that the statement in Theorem 1.1 holds.

Acknowledgments. Authors would like to express their sincere gratitude to one anonymous referee for his/her constructive comments for improving the quality of this paper.

\section{REFERENCES}

[1] M. Bramanti - L. Brandolini, $L^{p}$-estimates for nonvariational hypoelliptic operators with VMO coefficients, Trans. Amer. Math. Soc., 352 (2) (2000), pp. 781-822.

[2] M. Bramanti - L. Brandolini, Schauder estimates for parabolic nondivergence operators of Hörmande type, J. Differential Equations, 234 (1) (2007), pp. 177-245. 
[3] M. Bramanti - L. Brandolini, L ${ }^{p}$-estimates for uniformly hypoelliptic operators with discontinuous coefficients on homogenous groups, Rend. Sem. Mat. Univ. Politec. Torino, 58 (4) (2000), pp. 389-433.

[4] M. Bramanti - C. Cerutti, Commutators of singular integrals in homogeneous spaces, Boll. Unione Mat. Italiana, 10-B (7) (1996), pp. 843-883.

[5] M. Bramanti - C. CerutTi, $W_{p}^{1,2}$-solvability for the Cauchy-Dirichlet problem for parabolic equations with VMO coefficients, Comm. Partial Differential Equations, 18 (9-10) (1993), pp. 1735-1763.

[6] M. Bramanti, Commutators of integral operators with positive kernels, Le Mat., XLIX, 49 (1994), pp. 149-168.

[7] N. BuRGER, Espace des functions à variation moyenne bornée sur un espace de nature homogène, C.R. Math. Acad. Sci. Paris, 286 (1978), pp. 139-142.

[8] A. P. CALderón - A. Zygmund, Singular integral operators and differential equations, Amer. J. Math., 79 (1957), pp. 901-921.

[9] Y. Z. Chen - L. C. Wu, Elliptic equation of second order and elliptic system, Science Press, Beijing, 1991.

[10] F. ChIARENZA - M. FrasCA - P. Longo, Interior $W^{2, p}$-estimates for nondivergence elliptic equations with discontinuous coefficients, Ricerche Mat., 40 (1991), pp. 149-168.

[11] F. Chiarenza - M. Frasca - P. Longo, $W^{2, p}$-solvability of the Dirichlet problem for nondivergence form elliptic equations with VMO coefficients, Trans. Amer. Math. Soc., 336 (1993), pp. 841-853.

[12] R. Coifman - G. Weiss, Analyse Harmonique Non-Commutative sur Certains Espaces Homognes, in: Lecture Notes in Mathematics, Vol. 242 (Springer-Verlag, Berlin-Heidelberg-New York, 1971).

[13] G. Di FAzio - D .K. Palagachev - M. A. Ragusa, Global Morrey regularity of strong solutions to the Dirichlet problem for elliptic equations with discontinuous coefficients, J. Funct. Anal., 166 (2) (1999), pp. 179-196.

[14] G. Di FAZIO - M. A. RAGUSA, Interior estimates in Morrey spaces for strong solutions to nondivergence form elliptic equations with discontinuous coefficients, J. Funct. Anal., 112 (2) (1993), pp. 241-256.

[15] G. B. Folland, Subelliptic estimates and function spaces on nilpotent Lie groups, Ark. Mat., 13 (2) (1975), pp. 161-207.

[16] D. S. FAN - S. Z. LU - D. C. YANG, Boundedness of operators in Morrey spaces on homogenous spaces and its application, Acta. Math. Sinica (New Series), 14 (4) (1998), pp. 625-634.

[17] C. Fefferman - D. H. Phong, Subelliptic eigenvalue problems, in: Proceedings of the Conference on harmonic Analysis in honor of Antoni Zygmund, pp. 590-606, Wadsworth Math. Series, 1981.

[18] G. B. Folland - E. M. STEIN, Estimates for the $\bar{\partial}_{b}$ complex and analysis on the Heisenberg group, Comm. Pure Appl. Math., 27 (4) (1974), pp. 429-522.

[19] D. Gilbarg - N. S. Trudinger, Elliptic partial differential equations of second order, 2nd ed. Springer-Verlag, Berlin, 1983.

[20] L. HöRmander, Hypoelliptic second order differential equations, Acta Mathematica, 119 (1967), pp. 147-171.

[21] G. M. Lieberman, A mostly elementary proof of Morrey space estimates for elliptic and parabolic equations with VMO coefficients, J. Funct. Anal., 201 (2) (2003), pp. 457-479. 
[22] R. A. Macias - C. SEgovia, Lipschitz functions on spaces of homogeneous type, Adv. Math., 33 (3) (1979), pp. 257-270.

[23] A. NAgel - E. M. Stein - S. WAinger, Balls and metrics defined by vector fields I:Basic properties, Acta Math., 155 (1985), pp. 103-147.

[24] L. P. Rothschild - E. M. Stein, Hypoelliptic differential operators and nilpotent groups, Acta Math., 137 (1976), pp. 247-320.

[25] A. SancheZ-Calle, Fundamental solutions and geometry of sum of squares of vector fields, Invent. Math., 78 (1) (1984), pp. 143-160.

Manoscritto pervenuto in redazione il 22 marzo 2009. 
